\title{
Quantum M-wave and black 0-brane
}

\author{
Yoshifumi Hyakutake \\ Faculty of Science, Ibaraki University, \\ Bunkyo 2-1-1, Mito, Ibaraki 310-8512, Japan \\ E-mail: hyaku@mx.ibaraki.ac.jp
}

ABSTRACT: The effective action of superstring theory or M-theory is approximated by supergravity in the low energy limit, and quantum corrections to the supergravity are taken into account by including higher derivative terms. In this paper, we consider equations of motion with those higher derivative terms in M-theory and solve them to derive quantum M-wave solution. A quantum black 0-brane solution is also obtained by Kaluza-Klein dimensional reduction of the M-wave solution. The quantum black 0-brane is asymptotically flat and uniquely determined by imposing appropriate conditions. The mass and the R-R charge of the quantum black 0-brane are derived by using the ADM mass and the charge formulae, and we see that only the mass is affected by the quantum correction. Various limits of the quantum black 0-brane are also considered, and especially we show that an internal energy in the near horizon limit is correctly reproduced.

KeYwords: p-branes, Black Holes in String Theory, Black Holes

ARXIV EPRINT: 1407.6023 


\section{Contents}

1 Introduction 1

2 Review of classical M-wave and black 0-brane 3

3 Quantum M-wave and black 0-brane 5

3.1 Solve $E_{1}-E_{2}+E_{3}-E_{4}=0 \quad 7$

3.2 Solve $E_{2}+E_{3}=0 \quad 7$

3.3 Solve $E_{1}+E_{2}=0 \quad 9$

$\begin{array}{lll}3.4 & \text { Solve } E_{5}=0 & 10\end{array}$

$\begin{array}{ll}3.5 & \text { Solve } E_{1}=0 \\ 3.6 & 12\end{array}$

$\begin{array}{ll}3.6 & \text { Determination of integral constants } \\ \end{array}$

$4 \quad 3$ limits of the quantum black 0-brane $\quad 13$

$\begin{array}{ll}4.1 \text { The quantum black 0-brane solution } & 13\end{array}$

$\begin{array}{lll}4.2 & \text { The extremal limit } & 14\end{array}$

$\begin{array}{ll}4.3 & \text { The Schwarzschild limit } \\ \end{array}$

$\begin{array}{ll}4.4 & \text { The near horizon limit } \\ \end{array}$

5 Mass, R-R charge and internal energy of the quantum black 0-brane $\mathbf{1 5}$

$\begin{array}{ll}5.1 \text { The mass } & 16\end{array}$

$\begin{array}{ll}5.2 \text { The R-R charge } & 16\end{array}$

$\begin{array}{lll}6 & \text { Validity of the quantum black 0-brane solution } & 17\end{array}$

$\begin{array}{lll}7 & \text { Conclusion and discussion } & 17\end{array}$

A Higher derivative corrections to the ADM mass and the R-R charge 18

B Near horizon limit of the internal energy 22

\section{Introduction}

Superstring theory is a promising candidate for the theory of quantum gravity. UV divergence of the gravity is well controlled and it unifies gauge theory and gravity consistently. Therefore much effort has been devoted to reveal the quantum nature of the gravity. In this paper, we proceed those discussions and describe the quantum geometry in the superstring theory.

The superstring theory contains not only fundamental strings but also D $p$-branes which extend $p$ spacial directions [1]. In the low energy limit, the superstring theory is well 
approximated by the supergravity, and $\mathrm{D} p$-branes are described by a classical solution which is called a black $p$-brane $[2,3]$. In the extremal case where the mass and RamondRamond charge of the black $p$-brane are balanced, quantum corrections to the classical solution are suppressed. Then for a special class of black brane solutions, a statistical derivation of its entropy is possible from the dual gauge theory on the corresponding Dbranes [4]. Furthermore, by taking a near horizon limit of extremal black branes the geometry becomes anti-de-Sitter space-time, and it is conjectured that the superstring theory in the AdS background is dual to the gauge theory on the D-branes [5]. Correlation functions of the gauge theory can be calculated from the dual gravity theory $[6,7]$. On the other hand, the entropy counting or test of the gauge gravity duality of non-extremal black branes is quite difficult because quantum corrections become important which are not well understood so far. In this paper, we discuss quantum corrections to the non-extremal black 0-brane by explicitly solving equations of motion. ${ }^{1}$

In order to investigate quantum nature of the non-extremal black $p$-brane, we need to know quantum corrections to the supergravity. As the superstring theory is defined perturbatively, it is possible to derive corrections to the supergravity so as to be consistent with the scattering amplitude $[10,11]$ or $\sigma$-model calculations $[12,13]$. Among these corrections, the structure of higher curvature $R^{4}$ terms is well studied $[14,15]$ and they come from 4 gravitons amplitudes at tree and 1-loop levels $[10,11,16] . R^{4}$ terms are also derived by imposing local supersymmetry [17]-[22]. There are several attempts to solve the modified equations of motion [23]-[27]. For the black $p$-brane, however, it is difficult to solve the equations of motion consistently since the full form of the effective action including R-R gauge fields is not completely determined so far. ${ }^{2}$ In this paper we concentrate on the black 0-brane in type IIA supergravity, thus at least the knowledge of quantum corrections to a metric, a dilaton field and a R-R 1-form filed are necessary. This problem is resolved by noting that these fields are gathered into the metric in 11 dimensions [29]. Fortunately supersymmetric higher curvature corrections to the 11 dimensional supergravity are well known [20-22], so usual Kaluza-Klein dimensional reduction gives 1-loop quantum corrections to the type IIA supergravity which are relevant to the black 0-brane.

By taking account of the $R^{4}$ terms, quantum corrections to the near horizon geometry of the non-extremal black 0-brane is analytically solved in ref. [29]. This is quite useful to test the gauge gravity duality, since computer simulations of the dual gauge theory on the D0-branes are well developed in refs. [30]-[35]. In fact, the test of the gauge gravity duality is examined in ref. [36], including $1 / N^{2}$ quantum corrections to the classical gravity. And the analytic result from the gravity side is well reproduced from the gauge theory side numerically [36]. See also ref. [37] for introductory review.

In this paper, we generalize the discussions in ref. [29] to derive quantum corrections to asymptotically flat non-extremal M-wave and black 0-brane. We often call these solutions quantum M-wave and quantum black 0-brane. Organization of this paper is as follows. In section 2, we briefly review the classical M-wave and black 0-brane solution. The main

\footnotetext{
${ }^{1}$ Higher derivative corrections are also important for the extremal case [8, 9].

${ }^{2}$ In three dimensions the higher derivative corrections are well controlled. Although physical quantities are affected by the quantum corrections, the solution is the same as the classical one [28].
} 
part of this paper appears in section 3, where the quantum corrections to the non-extremal M-wave and black 0-brane are analytically solved. In section 4, we consider extremal limit, Schwarzschild one and near horizon one for the quantum black 0-brane. In section 5 , the ADM mass and the R-R charge of the quantum black 0-brane are derived. Validity of the solution is examined in section 6 , and section 7 is devoted to conclusion and discussion. In the appendix, we employ Noether and Wald's method to derive the quantum corrections to the ADM mass and the charge formulae. We also discuss the near horizon limit of the internal energy of the quantum black 0-brane.

\section{Review of classical M-wave and black 0-brane}

It is conjectured that the strong coupling limit of type IIA superstring theory in 10 dimensions is described by M-theory in 11 dimensions $[38,39]$. The string coupling constant $g_{s}$ and string length $\ell_{s}$ in the type IIA superstring theory are related to the radius of 11 th circle $R_{11}=g_{s} \ell_{s}$ and 11 dimensional Planck length $\ell_{p}=g_{s}^{1 / 3} \ell_{s}$ in the M-theory. A D0-brane is identified with a Kaluza-Klein mode, and the mass of the D0-brane is given by $1 / R_{11}$. In the low energy limit, the type IIA superstring theory and M-theory are approximated by type IIA supergravity and 11 dimensional supergravity, respectively. Then the D0-brane is approximated by a black 0-brane solution in the type IIA supergravity, and the Kaluza-Klein mode is described by a M-wave solution in the 11 dimensional supergravity. In this section, we briefly review classical properties of the black 0-brane via dimensional reduction of the M-wave solution. Simultaneously we fix the notations and conventions used in this paper.

The fields of the 11 dimensional supergravity consist of a graviton $g_{M N}$, a 3 -form field $A_{M N P}$ and a Majorana gravitino $\psi_{M}[40]$. Here $M, N, P$ are space-time indices in 11 dimensions, and both bosonic and fermionic fields have 128 physical degrees of freedom. Since we only consider the M-wave solution which does not couple to $A_{M N P}$ and $\psi_{M}$, a relevant part of the action is simply given by

$$
S_{11}^{(0)}=\frac{1}{2 \kappa_{11}^{2}} \int d^{11} x \sqrt{-g} R,
$$

where $2 \kappa_{11}^{2}=(2 \pi)^{8} \ell_{p}^{9}$. The equations of motion become $R_{M N}-\frac{1}{2} g_{M N} R=0$, and the following geometry becomes a solution.

$$
\begin{aligned}
d s_{11}^{2} & =-H^{-1} F d t^{2}+F^{-1} d r^{2}+r^{2} d \Omega_{8}^{2}+\left(H^{\frac{1}{2}} d z-\left(\frac{r_{+}}{r_{-}}\right)^{\frac{7}{2}} H^{-\frac{1}{2}} d t\right)^{2}, \\
H & =1+\frac{r_{-}^{7}}{r^{7}}, \quad F=1-\frac{r_{+}^{7}-r_{-}^{7}}{r^{7}} .
\end{aligned}
$$

This is the non-extremal M-wave solution, which contains two parameters $r_{ \pm}$. The extremal case is saturated when $r_{+}=r_{-}$, and Schwarzschild black hole smeared along $z$ direction is obtained when $r_{-} \rightarrow 0$.

The type IIA supergravity consists of a graviton $g_{\mu \nu}$, a dilaton $\phi$, a R-R 1-form field $C_{\mu}$, a NS-NS 2-form field $B_{\mu \nu}$, a R-R 3-form field $C_{\mu \nu \rho}$, a Majorana gravitino $\psi_{\mu}$ and a Majorana dilatino $\psi$. Here $\mu, \nu, \rho$ are space-time indices in 10 dimensions, and both bosonic 
and fermionic fields have the same physical degrees of freedom as those in 11 dimensional supergravity. The type IIA supergravity is derived by dimensional reduction of the 11 dimensional supergravity if we express the metric in 11 dimensions as

$$
g_{M N} d x^{M} d x^{N}=e^{-2 \phi / 3} g_{\mu \nu} d x^{\mu} d x^{\nu}+e^{4 \phi / 3}\left(d z-C_{\mu} d x^{\mu}\right)^{2},
$$

where $z$ direction is the circle with the radius $R_{11}$ [41]. By inserting this metric into the action (2.1), we obtain the 10 dimensional action of the form

$$
S_{10}^{(0)}=\frac{1}{2 \kappa_{10}^{2}} \int d^{10} x \sqrt{-g}\left\{e^{-2 \phi}\left(R+4 \partial_{\mu} \phi \partial^{\mu} \phi\right)-\frac{1}{4} G_{\mu \nu} G^{\mu \nu}\right\}
$$

where $2 \kappa_{10}^{2}=(2 \pi)^{7} \ell_{s}^{8} g_{s}^{2}$ and $G_{\mu \nu}$ is the field strength of $C_{\mu}$. Notice that the coordinate transformation $z^{\prime}=z+\chi(x)$ in 11 dimensions corresponds to the gauge transformation $C_{\mu}^{\prime}=C_{\mu}-\partial_{\mu} \chi$ in 10 dimensions.

The non-extremal black 0-brane solution is obtained by the dimensional reduction of the M-wave solution (2.2). By applying eq. (2.3), we obtain [42]

$$
d s_{10}^{2}=-H^{-\frac{1}{2}} F d t^{2}+H^{\frac{1}{2}} F^{-1} d r^{2}+H^{\frac{1}{2}} r^{2} d \Omega_{8}^{2}, \quad e^{\phi}=H^{\frac{3}{4}}, \quad C=\left(\frac{r_{+}}{r_{-}}\right)^{\frac{7}{2}} H^{-1} d t .
$$

Thus the purely geometrical object in 11 dimensions becomes the charged black hole in 10 dimensions. The event horizon is located at

$$
r_{H}=\left(r_{+}^{7}-r_{-}^{7}\right)^{\frac{1}{7}} \equiv r_{-} \alpha,
$$

where $\alpha$ is a dimensionless parameter. And the mass $M$ and the R-R charge $Q$ of the black 0 -brane are evaluated as

$$
M=\frac{V_{S^{8}}}{2 \kappa_{10}^{2}}\left(8 r_{+}^{7}-r_{-}^{7}\right), \quad Q=\frac{V_{S^{8}}}{2 \kappa_{10}^{2}} 7\left(r_{+} r_{-}\right)^{\frac{7}{2}} .
$$

$V_{S^{8}}=\frac{2 \pi^{9 / 2}}{\Gamma(9 / 2)}=\frac{2(2 \pi)^{4}}{7 \cdot 15}$ is the volume of $S^{8}$. Since the charge of $N$ D0-branes is quantized as $Q=\frac{N}{\ell_{s} g_{s}}$ in the type IIA superstring theory, the parameters $r_{ \pm}$can be expressed as

$$
r_{ \pm}^{7}=(1+\delta)^{ \pm 1}(2 \pi)^{2} 15 \pi g_{s} N \ell_{s}^{7}
$$

by introducing a non-negative parameter $\delta$. There are three limits of the solution (2.5) which are important in later sections. (See table. 1.) First one is the extremal limit $r_{+} \rightarrow r_{-}$which is equivalent to $\alpha \rightarrow 0$. Second one is the Schwarzschild limit $r_{-} \rightarrow 0$, where the charge $Q$ goes to zero. Final one is the near horizon limit which is realized by $r \rightarrow 0$ with $U \equiv r / \ell_{s}^{2}, \lambda \equiv g_{s} N /(2 \pi)^{2} \ell_{s}^{3}$ and $U_{0} \equiv r_{-} \alpha / \ell_{s}^{2}$ fixed [43]. This is equivalent to $\alpha \rightarrow 0$ by fixing $r_{-} \alpha / r, \ell_{s}^{2} /\left(r_{-} \alpha\right)$ and $g_{s}^{2} N^{2} /\left(r_{-} \alpha\right)^{3}$, so the near horizon limit corresponds to the near extremal limit. 


\begin{tabular}{|l|c|}
\hline Extremal limit & $\alpha \rightarrow 0$ \\
\hline Schwarzschild limit & $r_{-} \rightarrow 0$ \\
\hline Near horizon limit & $\alpha \rightarrow$ 0 by fixing $\frac{r_{-} \alpha}{r}, \frac{\ell_{s}^{2}}{r_{-} \alpha}$ and $\frac{g_{s}^{2} N^{2}}{\left(r_{-} \alpha\right)^{3}}$ \\
\hline
\end{tabular}

Table 1. Extremal, Schwarzschild and near horizon limits.

\section{Quantum M-wave and black 0-brane}

In this section, we consider leading quantum correction to the M-wave solution (2.2). Since the $\mathrm{M}$-wave solution is purely geometrical in 11 dimensions, the 3 -form $A_{M N P}$ is irrelevant to our analyses. Thus it is enough to investigate the effective action of the M-theory which depends only on higher curvature terms. The leading structure of those is known to be $R^{4}$ terms $[14,15]$. The explicit form of the effective action is given by

$$
\begin{aligned}
S_{11}=\frac{1}{2 \kappa_{11}^{2}} \int d^{11} x e\{R & \left.+\gamma\left(t_{8} t_{8} R^{4}-\frac{1}{4 !} \epsilon_{11} \epsilon_{11} R^{4}\right)\right\} \\
=\frac{1}{2 \kappa_{11}^{2}} \int d^{11} x e\{R & +24 \gamma\left(R_{a b c d} R_{a b c d} R_{e f g h} R_{e f g h}-64 R_{a b c d} R_{a e f g} R_{b c d h} R_{e f g h}\right. \\
& +2 R_{a b c d} R_{a b e f} R_{c d g h} R_{e f g h}+16 R_{a c b d} R_{a e b f} R_{c g d h} R_{e g f h} \\
& \left.\left.-16 R_{a b c d} R_{a e f g} R_{b e f h} R_{c d g h}-16 R_{a b c d} R_{a e f g} R_{b f e h} R_{c d g h}\right)\right\} .
\end{aligned}
$$

Although we neglected fermionic terms, a part of them is also obtained in refs. [20-22]. The expansion parameter in the action is given by

$$
\gamma=\frac{\pi^{2} \ell_{p}^{6}}{2^{11} 3^{2}}
$$

and $a, b, c, \cdots=0,1, \cdots, 10$ are local Lorentz indices. All indices are lowered for simplicity but should be contracted by the flat metric. Note that $\gamma \sim g_{s}^{2} \ell_{s}^{6}$, so when the effective action (3.1) is reduced to ten dimensions, it becomes one-loop leading corrections to the type IIA supergravity. By varying the effective action (3.1), equations of motion are obtained as

$$
\begin{aligned}
E_{i j} \equiv R_{i j}-\frac{1}{2} \eta_{i j} R+\gamma\{ & -\frac{1}{2} \eta_{i j}\left(t_{8} t_{8} R^{4}-\frac{1}{4 !} \epsilon_{11} \epsilon_{11} R^{4}\right) \\
& \left.+\frac{3}{2} R_{a b c i} X_{j}^{a b c}-\frac{1}{2} R_{a b c j} X_{i}^{a b c}-2 D_{(a} D_{b)} X_{i j}^{a}{ }_{i j}\right\}=0 .
\end{aligned}
$$

Here $D_{a}$ is a covariant derivative for local Lorentz indices and

$$
\begin{aligned}
X_{a b c d}=\frac{1}{2} & \left(X_{[a b][c d]}^{\prime}+X_{[c d][a b]}^{\prime}\right), \\
X_{a b c d}^{\prime}=96 & R_{a b c d} R_{e f g h} R_{e f g h}-16 R_{a b c e} R_{d f g h} R_{e f g h}+2 R_{a b e f} R_{c d g h} R_{e f g h} \\
& +16 R_{a e c g} R_{b f d h} R_{e f g h}-16 R_{a b e g} R_{c f e h} R_{d f g h}-16 R_{e f a g} R_{e f c h} R_{g b h d} \\
& \left.+8 R_{a b e f} R_{c e g h} R_{d f g h}\right) .
\end{aligned}
$$

The details of the derivation can be found in ref. [29]. 
Let us solve the eq. (3.3) up to the linear order of $\gamma$. The leading part of the metric (2.2) itself is not a solution of the eq. (3.3), we should relax the ansatz for the M-wave. Most general static ansatz with $\mathrm{SO}(9)$ rotation symmetry is given by

$$
\begin{aligned}
d s_{11}^{2} & =-H_{1}^{-1} F_{1} d t^{2}+F_{1}^{-1} d r^{2}+r^{2} d \Omega_{8}^{2}+\left(H_{2}^{\frac{1}{2}} d z-\sqrt{1+\alpha^{7}} H_{3}^{-\frac{1}{2}} d t\right)^{2}, \\
H_{i} & =1+\frac{r_{-}^{7}}{r^{7}}+\frac{\gamma}{r_{-}^{6} \alpha^{13}} h_{i}\left(\frac{r}{r_{-} \alpha}\right), \quad F_{1}=1-\frac{r_{-}^{7} \alpha^{7}}{r^{7}}+\frac{\gamma}{r_{-}^{6} \alpha^{6}} f_{1}\left(\frac{r}{r_{-} \alpha}\right) .
\end{aligned}
$$

Here $\alpha$ is given by $\alpha=\left(r_{+}^{7} / r_{-}^{7}-1\right)^{\frac{1}{7}}$, and $h_{i}(i=1,2,3)$ and $f_{1}$ are functions of $\frac{r}{r_{-} \alpha}$. Note that, up to the linear order of $\gamma$, the coordinate transformation $d z \rightarrow d z+c_{g} d t$ is interpreted as the change of $h_{2}(x)+h_{3}(x)$,

$$
\frac{\gamma}{r_{-}^{6} \alpha^{13}}\left(h_{2}+h_{3}\right) \rightarrow \frac{\gamma}{r_{-}^{6} \alpha^{13}}\left(h_{2}+h_{3}\right)+\frac{2 c_{g}}{\sqrt{1+\alpha^{2}}}\left(1+\frac{r_{-}^{7}}{r^{7}}\right)^{2} .
$$

It is clear that this corresponds to the gauge transformation of $C_{\mu}$ in 10 dimensions.

Now we insert the ansatz (3.5) into the eq. (3.3). In order to make the equations of motion simple, we introduce following dimensionless coordinates,

$$
\tau=\frac{t}{r_{-} \alpha}, \quad x=\frac{r}{r_{-} \alpha}, \quad y=\frac{z}{r_{-} \alpha} .
$$

Then all components of the metric are expressed as a function of $x$, and we obtain following 5 differential equations for $h_{i}(x)$ and $f_{1}(x) .^{3}$

$$
\begin{aligned}
E_{1}= & -7\left(9+32 \alpha^{7} x^{7}+16 \alpha^{14} x^{14}\right)\left(1+\alpha^{7} x^{7}\right) x^{34} f_{1}-\left(9+16 \alpha^{7} x^{7}\right)\left(1+\alpha^{7} x^{7}\right)^{2} x^{35} f_{1}^{\prime} \\
& -49\left(1+\alpha^{7}\right) x^{41} h_{1}+49\left(1-x^{7}\right) x^{34} h_{2}+\left(1-x^{7}\right)\left(23+16 \alpha^{7} x^{7}\right)\left(1+\alpha^{7} x^{7}\right) x^{35} h_{2}^{\prime} \\
& +2\left(1-x^{7}\right)\left(1+\alpha^{7} x^{7}\right)^{2} x^{36} h_{2}^{\prime \prime}+98\left(1+\alpha^{7}\right) x^{41} h_{3}+7\left(1+\alpha^{7}\right)\left(1+\alpha^{7} x^{7}\right) x^{42} h_{3}^{\prime} \\
& +17418240\left(61+\left(4032+4093 \alpha^{7}\right) x^{7}-3640\left(1+\alpha^{7}\right) x^{14}\right)\left(1+\alpha^{7} x^{7}\right)^{2}=0, \\
E_{2}= & 7\left(9+32 \alpha^{7} x^{7}+16 \alpha^{14} x^{14}\right)\left(1+\alpha^{7} x^{7}\right) x^{34} f_{1}+\left(9+16 \alpha^{7} x^{7}\right)\left(1+\alpha^{7} x^{7}\right)^{2} x^{35} f_{1}^{\prime} \\
& +7\left(9-\left(2-23 \alpha^{7}\right) x^{7}-16 \alpha^{7} x^{14}\right) x^{34} h_{1}+\left(1-x^{7}\right)\left(9+16 \alpha^{7} x^{7}\right)\left(1+\alpha^{7} x^{7}\right) x^{35} h_{1}^{\prime} \\
& -112\left(1-x^{7}\right)\left(1+\alpha^{7} x^{7}\right) x^{34} h_{2}-16\left(1-x^{7}\right)\left(1+\alpha^{7} x^{7}\right)^{2} x^{35} h_{2}^{\prime}-98\left(1+\alpha^{7}\right) x^{41} h_{3} \\
& -7\left(1+\alpha^{7}\right)\left(1+\alpha^{7} x^{7}\right) x^{42} h_{3}^{\prime}-17418240 \alpha^{34}\left(329+124 x^{7}\right)\left(1+\alpha^{7} x^{7}\right)^{3}=0, \\
E_{3}= & 7\left(19+24 \alpha^{7} x^{7}+12 \alpha^{14} x^{14}\right)\left(1+\alpha^{7} x^{7}\right) x^{34} f_{1}+7\left(5+4 \alpha^{7} x^{7}\right)\left(1+\alpha^{7} x^{7}\right)^{2} x^{35} f_{1}^{\prime} \\
& +2\left(1+\alpha^{7} x^{7}\right)^{3} x^{36} f_{1}^{\prime \prime}+14\left(6-5\left(4+3 \alpha^{7}\right) x^{7}+\alpha^{7} x^{14}\right) x^{34} h_{1} \\
& +7\left(4-\left(7+\alpha^{7}\right) x^{7}-2 \alpha^{7} x^{14}\right)\left(1+\alpha^{7} x^{7}\right) x^{35} h_{1}^{\prime}+2\left(1-x^{7}\right)\left(1+\alpha^{7} x^{7}\right)^{2} x^{36} h_{1}^{\prime \prime} \\
& -7\left(5-\left(26+23 \alpha^{7}\right) x^{7}+2 \alpha^{7} x^{14}\right) x^{34} h_{2}-7\left(3-\left(6+\alpha^{7}\right) x^{7}-2 \alpha^{7} x^{14}\right)\left(1+\alpha^{7} x^{7}\right) x^{35} h_{2}^{\prime} \\
& -2\left(1-x^{7}\right)\left(1+\alpha^{7} x^{7}\right)^{2} x^{36} h_{2}^{\prime \prime}+98\left(1+\alpha^{7}\right) x^{41} h_{3}+7\left(1+\alpha^{7}\right)\left(1+\alpha^{7} x^{7}\right) x^{42} h_{3}^{\prime} \\
& -30481920 \alpha^{34}\left(283-186 x^{7}\right)\left(1+\alpha^{7} x^{7}\right)^{3}=0, \\
E_{4}= & 7\left(37+32 \alpha^{7} x^{7}+16 \alpha^{14} x^{14}\right)\left(1+\alpha^{7} x^{7}\right) x^{34} f_{1}+\left(53+32 \alpha^{7} x^{7}\right)\left(1+\alpha^{7} x^{7}\right)^{2} x^{35} f_{1}^{\prime}
\end{aligned}
$$

\footnotetext{
${ }^{3}$ In order to derive the equations of motion, 3 independent Mathematica codes are used.
} 


$$
\begin{aligned}
& +2\left(1+\alpha^{7} x^{7}\right)^{3} x^{36} f_{1}^{\prime \prime}+147\left(1-\left(3+2 \alpha^{7}\right) x^{7}\right) x^{34} h_{1} \\
& +\left(37-\left(58+5 \alpha^{7}\right) x^{7}-16 \alpha^{7} x^{14}\right)\left(1+\alpha^{7} x^{7}\right) x^{35} h_{1}^{\prime}+2\left(1-x^{7}\right)\left(1+\alpha^{7} x^{7}\right)^{2} x^{36} h_{1}^{\prime \prime} \\
& +147\left(1+\alpha^{7}\right) x^{41} h_{2}+21\left(1+\alpha^{7}\right)\left(1+\alpha^{7} x^{7}\right) x^{42} h_{2}^{\prime} \\
& +294\left(1+\alpha^{7}\right) x^{41} h_{3}+21\left(1+\alpha^{7}\right)\left(1+\alpha^{7} x^{7}\right) x^{42} h_{3}^{\prime} \\
& -17418240\left(4093-\left(7672-61 \alpha^{7}\right) x^{7}+3640 x^{14}\right)\left(1+\alpha^{7} x^{7}\right)^{2}=0, \\
E_{5}= & 49 x^{34} h_{1}+7\left(1+\alpha^{7} x^{7}\right) x^{35} h_{1}^{\prime}+49 x^{34} h_{2}-\left(1+8 \alpha^{7} x^{7}\right)\left(1+\alpha^{7} x^{7}\right) x^{35} h_{2}^{\prime} \\
& -\left(1+\alpha^{7} x^{7}\right)^{2} x^{36} h_{2}^{\prime \prime}-98 x^{34} h_{3}-2\left(11+15 \alpha^{7} x^{7}+4 \alpha^{14} x^{14}\right) x^{35} h_{3}^{\prime} \\
& -\left(1+\alpha^{7} x^{7}\right)^{2} x^{36} h_{3}^{\prime \prime}+975421440 \alpha^{27}\left(72-65 x^{7}\right)\left(1+\alpha^{7} x^{7}\right)^{2}=0 .
\end{aligned}
$$

The above equations come from the linear order of $\gamma$, and equations of $\mathcal{O}\left(\gamma^{0}\right)$ are automatically satisfied. In following subsections, we will solve 5 equations of motion step by step and determine $h_{i}(x)$ and $f_{1}(x)$. Although there appear several integral constants, these are uniquely fixed by imposing appropriate conditions. For example, we require that $h_{i}(x)$ and $f_{1}(x)$ do not diverge around the event horizon, and $h_{3}(x)$ should be determined up to the gauge transformation (3.6). Consistency conditions with the extremal limit and the Schwarzschild one in table 1 are also taken into account. A complete form of the solution is summarized in section 4 .

\subsection{Solve $E_{1}-E_{2}+E_{3}-E_{4}=0$}

First let us consider a combination of $E_{1}-E_{2}+E_{3}-E_{4}$. After some calculations, it is simplified as

$$
\begin{aligned}
0 & =\frac{E_{1}-E_{2}+E_{3}-E_{4}}{36 x^{28}\left(1+\alpha^{7} x^{7}\right)^{3}} \\
& =-\left(x^{7} f_{1}\right)^{\prime}-\frac{1-x^{7}}{2}\left\{\frac{x^{7}\left(h_{1}-h_{2}\right)}{1+\alpha^{7} x^{7}}\right\}^{\prime}+\frac{362880}{x^{28}}\left(5317-4254 x^{7}\right) .
\end{aligned}
$$

From this equation, $\left(x^{7} f_{1}\right)^{\prime}$ is expressed in terms of $\left(h_{1}-h_{2}\right)$ as

$$
\left(x^{7} f_{1}\right)^{\prime}=-\frac{1-x^{7}}{2}\left\{\frac{x^{7}\left(h_{1}-h_{2}\right)}{1+\alpha^{7} x^{7}}\right\}^{\prime}+\frac{362880}{x^{28}}\left(5317-4254 x^{7}\right) .
$$

There remain 4 equations to be solved.

\subsection{Solve $E_{2}+E_{3}=0$}

Next we evaluate a combination of $E_{2}+E_{3}$.

$$
\begin{aligned}
0= & \frac{E_{2}+E_{3}}{\left(1+\alpha^{7} x^{7}\right)^{3}} \\
= & 2 x^{28}\left\{8\left(x^{7} f_{1}\right)^{\prime}+x\left(x^{7} f_{1}\right)^{\prime \prime}\right\}+49\left(3-\left(6+\alpha^{7}\right) x^{7}-2 \alpha^{7} x^{14}\right) \frac{x^{34}\left(h_{1}-h_{2}\right)}{\left(1+\alpha^{7} x^{7}\right)^{3}} \\
& +\left(37-\left(58-9 \alpha^{7}\right) x^{7}-30 \alpha^{7} x^{14}\right) \frac{x^{35}\left(h_{1}-h_{2}\right)^{\prime}}{\left(1+\alpha^{7} x^{7}\right)^{2}}+2\left(1-x^{7}\right) \frac{x^{36}\left(h_{1}-h_{2}\right)^{\prime \prime}}{1+\alpha^{7} x^{7}}
\end{aligned}
$$




$$
\begin{aligned}
& -4354560\left(3297-806 x^{7}\right) \\
= & 2 x^{28}\left\{8\left(x^{7} f_{1}\right)^{\prime}+x\left(x^{7} f_{1}\right)^{\prime \prime}\right\}+3\left(3-10 x^{7}\right) x^{28}\left\{\frac{x^{7}\left(h_{1}-h_{2}\right)}{1+\alpha^{7} x^{7}}\right\}^{\prime} \\
& +2\left(1-x^{7}\right) x^{29}\left\{\frac{x^{7}\left(h_{1}-h_{2}\right)}{1+\alpha^{7} x^{7}}\right\}^{\prime \prime}-4354560\left(3297-806 x^{7}\right) \\
= & \left(1-x^{7}\right) x^{29}\left\{\frac{x^{7}\left(h_{1}-h_{2}\right)}{1+\alpha^{7} x^{7}}\right\}^{\prime \prime}+\left(1-15 x^{7}\right) x^{28}\left\{\frac{x^{7}\left(h_{1}-h_{2}\right)}{1+\alpha^{7} x^{7}}\right\}^{\prime} \\
& -1451520\left(63061-30069 x^{7}\right) \\
= & \frac{x^{28}}{1-x^{7}}\left[x\left(1-x^{7}\right)^{2}\left\{\frac{x^{7}\left(h_{1}-h_{2}\right)}{1+\alpha^{7} x^{7}}\right\}^{\prime}\right]^{\prime}-1451520\left(63061-30069 x^{7}\right) .
\end{aligned}
$$

Note that the eq. (3.14) is employed to eliminate $f_{1}(x)$ out of the equation. It is possible to integrate the above equation once, and the result becomes

$$
\begin{aligned}
\left\{\frac{x^{7}\left(h_{1}-h_{2}\right)}{1+\alpha^{7} x^{7}}\right\}^{\prime}= & \frac{1451520}{\left(1-x^{7}\right)^{2}}\left(-\frac{63061}{27 x^{28}}+\frac{9313}{2 x^{21}}-\frac{2313}{x^{14}}\right)+\frac{c_{1}}{x\left(1-x^{7}\right)^{2}} \\
= & 1451520\left\{-\frac{63061}{27 x^{28}}-\frac{793}{54 x^{21}}-\frac{61}{9 x^{14}}+\frac{61}{54 x^{7}}+\frac{61\left(8-x^{7}\right)}{54\left(1-x^{7}\right)^{2}}\right\} \\
& +\frac{c_{1}}{x\left(1-x^{7}\right)^{2}},
\end{aligned}
$$

where $c_{1}$ is an integral constant. $h_{1}(x)-h_{2}(x)$ can be derived by integrating the eq. (3.16). In order to execute the integral, we define the following function,

$$
\begin{aligned}
I(x)= & \log \frac{x^{7}(x-1)}{x^{7}-1}-\sum_{n=1,3,5} \cos \frac{n \pi}{7} \log \left(x^{2}+2 x \cos \frac{n \pi}{7}+1\right) \\
& -2 \sum_{n=1,3,5} \sin \frac{n \pi}{7}\left\{\tan ^{-1}\left(\frac{x+\cos \frac{n \pi}{7}}{\sin \frac{n \pi}{7}}\right)-\frac{\pi}{2}\right\} .
\end{aligned}
$$

It is useful to note following relations.

$$
\begin{array}{r}
\left\{\log \left(1-\frac{1}{x^{7}}\right)\right\}^{\prime}=-\frac{7}{x\left(1-x^{7}\right)}, \\
\left\{\frac{1}{7} I(x)+\frac{1}{7} \log \left(1-\frac{1}{x^{7}}\right)\right\}^{\prime}=-\frac{1}{\left.1-x^{7}\right)}, \\
\left\{\frac{1}{7} \frac{x}{x^{7}-1}+\frac{6}{49} I(x)+\frac{6}{49} \log \left(1-\frac{1}{x^{7}}\right)\right\}^{\prime}=-\frac{1}{\left(1-x^{7}\right)^{2}}, \\
\left\{\frac{1}{7} \frac{1}{x^{7}-1}+\frac{1}{7} \log \left(1-\frac{1}{x^{7}}\right)\right\}^{\prime}=-\frac{1}{x\left(1-x^{7}\right)^{2}} .
\end{array}
$$

By using these relations it is possible to integrate the eq. (3.16), and the result becomes

$$
\begin{aligned}
\frac{h_{1}-h_{2}}{1+\alpha^{7} x^{7}}= & \frac{1130053120}{9 x^{34}}+\frac{1065792}{x^{27}}+\frac{9838080}{13 x^{20}}-\frac{273280}{x^{13}}-\frac{1639680\left(x-\tilde{c}_{1}\right)}{x^{7}\left(x^{7}-1\right)} \\
& -\frac{1639680}{x^{7}} I(x)+\frac{c_{2}}{x^{7}}-\left(1-\tilde{c}_{1}\right) \frac{1639680}{x^{7}} \log \left(1-\frac{1}{x^{7}}\right)
\end{aligned}
$$


where $c_{2}$ is an integral constant and $c_{1}$ is redefined as $c_{1}=-11477760 \tilde{c}_{1}$. Because $h_{1}(x)-$ $h_{2}(x)$ should be finite at $x=1$, we choose $\tilde{c}_{1}=1$. Then $h_{1}(x)-h_{2}(x)$ takes the form of

$$
\begin{aligned}
& h_{1}-h_{2}=\frac{1130053120}{9 x^{34}}+\frac{448\left(21411+2522440 \alpha^{7}\right)}{9 x^{27}}+\frac{81984\left(120+169 \alpha^{7}\right)}{13 x^{20}} \\
& -\frac{273280\left(13-36 \alpha^{7}\right)}{13 x^{13}}-\frac{1639680}{x^{7}}+\frac{273280\left(6-\alpha^{7}\right)}{x^{6}} \\
& -\frac{1639680\left(1+\alpha^{7}\right)(x-1)}{x^{7}-1}-1639680\left(\frac{1}{x^{7}}+\alpha^{7}\right) I(x)+\frac{c_{2}}{x^{7}}+c_{2} \alpha^{7} .
\end{aligned}
$$

Now we are ready to derive an explicit form of $f_{1}(x)$. By inserting the eq. (3.16) into the eq. (3.14), we obtain differential equation for $f_{1}(x)$,

$$
\begin{aligned}
\left(x^{7} f_{1}\right)^{\prime}= & 725760\left\{\left(1-x^{7}\right)\left(\frac{63061}{27 x^{28}}+\frac{793}{54 x^{21}}+\frac{61}{9 x^{14}}-\frac{61}{54 x^{7}}\right)-\frac{61\left(8-x^{7}\right)}{54\left(1-x^{7}\right)}\right\} \\
& +\frac{5738880}{x\left(1-x^{7}\right)}+\frac{362880}{x^{28}}\left(5317-4254 x^{7}\right) \\
= & 725760\left(\frac{269681}{54 x^{28}}-\frac{240187}{54 x^{21}}-\frac{427}{54 x^{14}}-\frac{427}{54 x^{7}}\right)+\frac{5738880(1-x)}{x\left(1-x^{7}\right)}
\end{aligned}
$$

By using the relations (3.18), $f_{1}(x)$ can be solved as

$$
f_{1}=-\frac{1208170880}{9 x^{34}}+\frac{161405664}{x^{27}}+\frac{5738880}{13 x^{20}}+\frac{956480}{x^{13}}+\frac{c_{3}}{x^{7}}+\frac{819840}{x^{7}} I(x),
$$

where $c_{3}$ is an integral constant. So far we solved $E_{1}-E_{2}+E_{3}-E_{4}=0$ and $E_{2}+E_{3}=0$ to obtain $h_{1}(x)-h_{2}(x)$ and $f_{1}(x)$. There remain 3 equations to be solved.

\subsection{Solve $E_{1}+E_{2}=0$}

A linear combination of $E_{1}+E_{2}$ is calculated as follows.

$$
\begin{aligned}
0= & -\frac{E_{1}+E_{2}}{2 x^{28}\left(1-x^{7}\right)\left(1+\alpha^{7} x^{7}\right)^{2}} \\
= & -\frac{9+16 \alpha^{7} x^{7}}{2}\left\{\frac{x^{7}\left(h_{1}-h_{2}\right)}{1+\alpha^{7} x^{7}}\right\}^{\prime}+\frac{34836480}{x^{28}}\left(67-\left(910+941 \alpha^{7}\right) x^{7}\right)-\left(x^{8} h_{2}^{\prime}\right)^{\prime} \\
= & 725760\left(9+16 \alpha^{7} x^{7}\right)\left\{\frac{63061}{27 x^{28}}+\frac{793}{54 x^{21}}+\frac{61}{9 x^{14}}-\frac{61}{54 x^{7}}-\frac{61\left(8-x^{7}\right)}{54\left(1-x^{7}\right)^{2}}\right\} \\
& +\frac{5738880\left(9+16 \alpha^{7} x^{7}\right)}{x\left(1-x^{7}\right)^{2}}+\frac{34836480}{x^{28}}\left(67-\left(910+941 \alpha^{7}\right) x^{7}\right)-\left(x^{8} h_{2}^{\prime}\right)^{\prime} \\
= & 725760\left(\frac{72709}{3 x^{28}}-\frac{2351583+421120 \alpha^{7}}{54 x^{21}}+\frac{1647+6344 \alpha^{7}}{27 x^{14}}-\frac{183-1952 \alpha^{7}}{18 x^{7}}\right) \\
& +819840\left(9+16 \alpha^{7}\right)\left\{\frac{7(1-x)}{x\left(1-x^{7}\right)^{2}}-\frac{1}{1-x^{7}}\right\}-\frac{91822080 \alpha^{7}(1-x)}{x\left(1-x^{7}\right)}-\left(x^{8} h_{2}^{\prime}\right)^{\prime} .
\end{aligned}
$$

Here we employed the eq. (3.16). This is a differential equation only on $h_{2}(x)$, and it is possible to integrate it once by employing the relations (3.18). The result is calculated as

$$
h_{2}^{\prime}=-\frac{651472640}{x^{35}}+\frac{672\left(2351583+421120 \alpha^{7}\right)}{x^{28}}-\frac{1639680\left(27+104 \alpha^{7}\right)}{13 x^{21}}
$$




$$
\begin{aligned}
& +\frac{409920\left(3-32 \alpha^{7}\right)}{x^{14}}+819840\left(9+16 \alpha^{7}\right) \frac{1-x}{x^{8}\left(1-x^{7}\right)}+\frac{7378560}{x^{8}} I(x)-\frac{7 c_{4}}{x^{8}} \\
= & -\frac{651472640}{x^{35}}+\frac{672\left(2351583+421120 \alpha^{7}\right)}{x^{28}}-\frac{1639680\left(27+104 \alpha^{7}\right)}{13 x^{21}} \\
& +\frac{409920\left(3-32 \alpha^{7}\right)}{x^{14}}+1639680\left(9+8 \alpha^{7}\right) \frac{1-x}{x^{8}\left(1-x^{7}\right)}+\left(-\frac{1054080}{x^{7}} I(x)\right)^{\prime}-\frac{7 c_{4}}{x^{8}} \\
= & -\frac{651472640}{x^{35}}+\frac{672\left(2351583+421120 \alpha^{7}\right)}{x^{28}}-\frac{1639680\left(27+104 \alpha^{7}\right)}{13 x^{21}} \\
& +\frac{409920\left(3-32 \alpha^{7}\right)}{x^{14}}+\frac{1639680\left(9+8 \alpha^{7}\right)}{x^{8}}-\frac{1639680\left(9+8 \alpha^{7}\right)}{x^{7}} \\
& +\left\{1054080\left(2-\frac{1}{x^{7}}+\frac{16 \alpha^{7}}{9}\right) I(x)\right\}^{\prime}-\frac{7 c_{4}}{x^{8}},
\end{aligned}
$$

where $c_{4}$ is an integral constant. Now it is easy to integrate the above differential equation, and $h_{2}(x)$ is derived as

$$
\begin{aligned}
h_{2}= & \frac{19160960}{x^{34}}-\frac{224\left(2351583+421120 \alpha^{7}\right)}{9 x^{27}}+\frac{81984\left(27+104 \alpha^{7}\right)}{13 x^{20}} \\
& -\frac{409920\left(3-32 \alpha^{7}\right)}{13 x^{13}}-\frac{234240\left(9+8 \alpha^{7}\right)}{x^{7}}+\frac{273280\left(9+8 \alpha^{7}\right)}{x^{6}} \\
& +1054080\left(2-\frac{1}{x^{7}}+\frac{16 \alpha^{7}}{9}\right) I(x)+\frac{c_{4}}{x^{7}}+c_{5},
\end{aligned}
$$

where $c_{5}$ is an integral constant. Inserting this result into the eq. (3.20), we obtain

$$
\begin{aligned}
h_{1}= & \frac{1302501760}{9 x^{34}}-\frac{224\left(2308761-4623760 \alpha^{7}\right)}{9 x^{27}}+\frac{1721664\left(7+13 \alpha^{7}\right)}{13 x^{20}} \\
& -\frac{956480\left(5-24 \alpha^{7}\right)}{13 x^{13}}-\frac{1873920\left(2+\alpha^{7}\right)}{x^{7}}+\frac{273280\left(15+7 \alpha^{7}\right)}{x^{6}} \\
& -1639680\left(1+\alpha^{7}\right) \frac{x-1}{x^{7}-1}+117120\left(18-\frac{23}{x^{7}}+2 \alpha^{7}\right) I(x) \\
& +\frac{c_{2}+c_{4}}{x^{7}}+c_{2} \alpha^{7}+c_{5} .
\end{aligned}
$$

We have already solved $E_{1}-E_{2}+E_{3}-E_{4}=0, E_{2}+E_{3}=0$ and $E_{1}+E_{2}=0$ to obtain $h_{1}(x), h_{2}(x)$ and $f_{1}(x)$. There remain 2 equations to be solved.

\subsection{Solve $E_{5}=0$}

The equation $E_{5}=0$ should be solved to determine $h_{3}(x)$.

$$
\begin{aligned}
0 & =\frac{E_{5}}{7 x^{28}\left(1+\alpha^{7} x^{7}\right)^{2}} \\
& =\left\{-\frac{2 x^{7}\left(h_{2}+h_{3}\right)}{1+\alpha^{7} x^{7}}-\frac{x^{8}}{7}\left(h_{2}+h_{3}\right)^{\prime}+\frac{x^{7}\left(h_{1}+3 h_{2}\right)}{1+\alpha^{7} x^{7}}-\frac{371589120}{x^{27}}+\frac{452874240}{x^{20}}\right\}^{\prime} \\
& =\left[-\frac{\left(1+\alpha^{7} x^{7}\right)^{2}}{7 x^{6}}\left\{\frac{x^{14}\left(h_{2}+h_{3}\right)}{\left(1+\alpha^{7} x^{7}\right)^{2}}\right\}^{\prime}+\frac{x^{7}\left(h_{1}+3 h_{2}\right)}{1+\alpha^{7} x^{7}}-\frac{371589120}{x^{27}}+\frac{452874240}{x^{20}}\right]^{\prime}
\end{aligned}
$$


This can be easily integrated once, and by using the eqs. (3.25) and (3.26) we obtain a differential equation for $h_{2}(x)+h_{3}(x)$.

$$
\begin{aligned}
&\{\left.\frac{x^{14}\left(h_{2}+h_{3}\right)}{\left(1+\alpha^{7} x^{7}\right)^{2}}\right\}^{\prime} \\
&= \frac{7 x^{13}}{\left(1+\alpha^{7} x^{7}\right)^{3}}\left\{h_{1}+3 h_{2}-\frac{371589120\left(1+\alpha^{7} x^{7}\right)}{x^{34}}+\frac{452874240\left(1+\alpha^{7} x^{7}\right)}{x^{27}}+\frac{c_{6}\left(1+\alpha^{7} x^{7}\right)}{x^{7}}\right\} \\
&= \frac{7 x^{13}}{\left(1+\alpha^{7} x^{7}\right)^{3}}\left\{-\frac{1524454400}{9 x^{34}}+\frac{2240\left(883233-1156952 \alpha^{7}\right)}{9 x^{27}}+\frac{4032\left(4636+1472055 \alpha^{7}\right)}{13 x^{20}}\right. \\
&-\frac{273280\left(31-228 \alpha^{7}\right)}{13 x^{13}}-\frac{234240\left(43+32 \alpha^{7}\right)}{x^{7}}+\frac{273280\left(42+31 \alpha^{7}\right)}{x^{6}}-\frac{1639680\left(1+\alpha^{7}\right)(x-1)}{x^{7}-1} \\
&\left.+234240\left(36-\frac{25}{x^{7}}+25 \alpha^{7}\right) I(x)+\frac{c_{2}+4 c_{4}+c_{6}}{x^{7}}+c_{2} \alpha^{7}+4 c_{5}+c_{6} \alpha^{7}\right\} \\
&= {\left[\frac { x ^ { 1 4 } } { ( 1 + \alpha ^ { 7 } x ^ { 7 } ) ^ { 2 } } \left\{\frac{533559040}{9 x^{34}}-\frac{118368320}{x^{27}}-\frac{21807744}{13 x^{20}}+\frac{3747840}{x^{14}}-\frac{59301760}{13 x^{13}}-\frac{4216320}{x^{7}}\right.\right.} \\
&\left.\left.\quad+\frac{4919040}{x^{6}}+234240\left(18-\frac{25}{x^{7}}\right) I(x)+\frac{c_{2}+4 c_{4}+c_{6}}{x^{7}}+c_{2} \alpha^{7}+2 c_{4} \alpha^{7}+2 c_{5}+c_{6} \alpha^{7}\right\}\right]^{\prime}
\end{aligned}
$$

where $c_{6}$ is an integral constant. In order to obtain the last equality we used relations (3.18). This is a differential equation only on $h_{2}(x)+h_{3}(x)$ and we obtain

$$
\begin{aligned}
h_{2}+h_{3}= & \frac{533559040}{9 x^{34}}-\frac{118368320}{x^{27}}-\frac{21807744}{13 x^{20}}+\frac{3747840}{x^{14}} \\
& -\frac{59301760}{13 x^{13}}-\frac{4216320}{x^{7}}+\frac{4919040}{x^{6}}+234240\left(18-\frac{25}{x^{7}}\right) I(x) \\
& +\frac{c_{2}+4 c_{4}+c_{6}}{x^{7}}+c_{2} \alpha^{7}+2 c_{4} \alpha^{7}+2 c_{5}+c_{6} \alpha^{7}+c_{g}\left(\alpha^{7}+\frac{1}{x^{7}}\right)^{2},
\end{aligned}
$$

where $c_{g}$ is an integral constant. As explained in eq. (3.6), $c_{g}$ corresponds to the parameter of the coordinate transformation $d z \rightarrow d z+c_{g} d t$. Since the explicit form of $h_{2}(x)$ is given by the eq. (3.25), $h_{3}(x)$ is expressed as

$$
\begin{aligned}
h_{3}= & \frac{361110400}{9 x^{34}}-\frac{224\left(2404287-421120 \alpha^{7}\right)}{9 x^{27}}-\frac{81984\left(293+104 \alpha^{7}\right)}{13 x^{20}} \\
& +\frac{3747840}{x^{14}}-\frac{136640\left(425+96 \alpha^{7}\right)}{13 x^{13}}-\frac{234240\left(9-8 \alpha^{7}\right)}{x^{7}} \\
& +\frac{273280\left(9-8 \alpha^{7}\right)}{x^{6}}+117120\left(18-\frac{41}{x^{7}}-16 \alpha^{7}\right) I(x) \\
& +\frac{c_{2}+3 c_{4}+c_{6}}{x^{7}}+c_{2} \alpha^{7}+2 c_{4} \alpha^{7}+c_{5}+c_{6} \alpha^{7}+c_{g}\left(\alpha^{7}+\frac{1}{x^{7}}\right)^{2} .
\end{aligned}
$$

Now we have solved 4 equations and derived $h_{i}(x)$ and $f_{1}(x)$. There remain only one equation to be solved. 


\subsection{Solve $E_{1}=0$}

The final independent equation which should be solved is $E_{1}=0$. By inserting solutions obtained so far, the equation $E_{1}=0$ gives a relation among integral constants.

$$
-\frac{E_{1}}{49 \alpha^{7} x^{34}\left(1+x^{7}\right)}=1873920 \alpha^{14}+c_{3} \alpha^{7}-c_{4}\left(2+\alpha^{7}\right)-c_{5}-c_{6}\left(1+\alpha^{7}\right)=0 .
$$

From this, $c_{3}$ is expressed as

$$
c_{3} \alpha^{7}=-1873920 \alpha^{14}+c_{4}\left(2+\alpha^{7}\right)+c_{5}+c_{6}\left(1+\alpha^{7}\right) .
$$

There remain 4 integral constants $c_{2}, c_{4}, c_{5}$ and $c_{6}$ in $h_{i}(x)$, which should be fixed by imposing boundary conditions and requiring consistencies with various limits in table 1.

\subsection{Determination of integral constants}

Our remaining task is to determine integral constants $c_{2}, c_{4}, c_{5}$ and $c_{6}$ in $h_{i}(x)$. First of all, the solution should be asymptotically flat. This means that when $x$ goes to the infinity, $h_{1}(x), h_{2}(x)$ and $f_{1}(x)$ should vanish and $h_{2}(x)+h_{3}(x)$ should do up to the coordinate transformation (3.6). Since the function $I(x)$ is expanded around $x \sim \infty$ as

$$
I(x)=-\frac{7}{6 x^{6}}+\frac{1}{x^{7}}-\frac{7}{13 x^{13}}+\frac{1}{2 x^{14}}+\mathcal{O}\left(\frac{1}{x^{15}}\right),
$$

asymptotic behaviors of $h_{1}(x), h_{2}(x), h_{2}(x)+h_{3}(x)$ and $f_{1}(x)$ are evaluated like

$$
\begin{aligned}
h_{1} & =c_{2} \alpha^{7}+c_{5}+\frac{c_{2}+c_{4}}{x^{7}}+\frac{1756800 \alpha^{7}}{x^{14}}+\mathcal{O}\left(\frac{1}{x^{15}}\right), \\
h_{2} & =c_{5}+\frac{c_{4}}{x^{7}}+\frac{936960 \alpha^{7}}{x^{14}}+\mathcal{O}\left(\frac{1}{x^{15}}\right), \\
h_{2}+h_{3} & =c_{2} \alpha^{7}+2 c_{5}+c_{6} \alpha^{7}+\frac{c_{2}+c_{6}}{x^{7}}-\frac{2 c_{4}}{\alpha^{7} x^{14}}+\left(c_{g}+\frac{2 c_{4}}{\alpha^{7}}\right)\left(\alpha^{7}+\frac{1}{x^{7}}\right)^{2}+\mathcal{O}\left(\frac{1}{x^{15}}\right), \\
f_{1} & =\frac{c_{3}}{x^{7}}+\frac{819840}{x^{14}}+\mathcal{O}\left(\frac{1}{x^{15}}\right) .
\end{aligned}
$$

Due to the asymptotic flatness, constants in $h_{1}(x)$ and $h_{2}(x)$ should vanish in the above equations. Therefore we obtain

$$
c_{2}=c_{5}=0,
$$

and asymptotic behaviors of $h_{1}(x), h_{2}(x), h_{2}(x)+h_{3}(x)$ and $f_{1}(x)$ become

$$
\begin{aligned}
h_{1} & =\frac{c_{4}}{x^{7}}+\frac{1756800 \alpha^{7}}{x^{14}}+\mathcal{O}\left(\frac{1}{x^{15}}\right), \\
h_{2} & =\frac{c_{4}}{x^{7}}+\frac{936960 \alpha^{7}}{x^{14}}+\mathcal{O}\left(\frac{1}{x^{15}}\right), \\
h_{2}+h_{3} & =-\frac{c_{6}}{x^{7}}-\frac{2 c_{4}+c_{6}}{\alpha^{7} x^{14}}+\left(c_{g}+\frac{2 c_{4}+c_{6}}{\alpha^{7}}\right)\left(\alpha^{7}+\frac{1}{x^{7}}\right)^{2}+\mathcal{O}\left(\frac{1}{x^{15}}\right),
\end{aligned}
$$




$$
f_{1}=\frac{c_{3}}{x^{7}}+\frac{819840}{x^{14}}+\mathcal{O}\left(\frac{1}{x^{15}}\right)
$$

Note that $f_{1}(x)$ automatically goes to zero when $x \rightarrow \infty$.

Below we consider consistency conditions with the extremal limit and the Schwarzschild one in the table 1 . In the extremal limit $\alpha \rightarrow 0$, the classical geometry preserves a half supersymmetry and does not receive any higher curvature corrections [44]. Therefore the mass and the charge are fixed to be the same, and we require $\frac{1}{\alpha^{6}} f_{1}\left(\frac{r}{r_{-} \alpha}\right)$ should be zero and $\frac{1}{\alpha^{13}}\left(h_{2}\left(\frac{r}{r_{-} \alpha}\right)+h_{3}\left(\frac{r}{r_{-} \alpha}\right)\right)$ should vanish up to the gauge transformation. These conditions are satisfied if $c_{3} \sim \alpha^{n}(n \geq 0)$ and $c_{4} \sim c_{6} \sim \alpha^{n}(n \geq 7)$.

On the other hand, by taking the Schwarzschild limit $r_{-} \rightarrow 0$ with $r_{-} \alpha$ fixed, $f_{1}\left(\frac{r}{r_{-}}\right)$ should be finite and $r_{-}^{7}\left(h_{2}\left(\frac{r}{r_{-} \alpha}\right)+h_{3}\left(\frac{r}{r_{-} \alpha}\right)\right)$ should vanish up to the gauge transformation. These conditions are satisfied if $c_{3} \sim \alpha^{n}(n \leq 0), c_{4} \sim \alpha^{n}(n \leq 13)$ and $c_{6} \sim \alpha^{n}(n \leq 6)$. Combining these restrictions with the eq. (3.31), we obtain

$$
c_{3}=3747840, \quad c_{4}=1873920 \alpha^{7}, \quad c_{6}=0,
$$

and finally asymptotic behaviors of $h_{1}(x), h_{2}(x), h_{2}(x)+h_{3}(x)$ and $f_{1}(x)$ become

$$
\begin{aligned}
h_{1} & =\frac{1873920 \alpha^{7}}{x^{7}}+\frac{1756800 \alpha^{7}}{x^{14}}+\mathcal{O}\left(\frac{1}{x^{15}}\right), \\
h_{2} & =\frac{1873920 \alpha^{7}}{x^{7}}+\frac{936960 \alpha^{7}}{x^{14}}+\mathcal{O}\left(\frac{1}{x^{15}}\right), \\
h_{2}+h_{3} & =-\frac{3747840}{x^{14}}+\left(c_{g}+3747840\right)\left(\alpha^{7}+\frac{1}{x^{7}}\right)^{2}+\mathcal{O}\left(\frac{1}{x^{15}}\right), \\
f_{1} & =\frac{3747840}{x^{7}}+\frac{819840}{x^{14}}+\mathcal{O}\left(\frac{1}{x^{15}}\right) .
\end{aligned}
$$

Therefore all integral constants except $c_{g}$ are uniquely determined by imposing asymptotic flatness and requiring consistencies with the extremal limit and the Schwarzschild one.

\section{3 limits of the quantum black 0-brane}

\subsection{The quantum black 0-brane solution}

Let us summarize the quantum black 0-brane solution. The dimensional reduction of the quantum M-wave solution is identified with the quantum black 0-brane in 10 dimensions, and the geometry is described by

$$
\begin{aligned}
d s_{10}^{2} & =-H_{1}^{-1} H_{2}^{\frac{1}{2}} F_{1} d t^{2}+H_{2}^{\frac{1}{2}} F_{1}^{-1} d r^{2}+H_{2}^{\frac{1}{2}} r^{2} d \Omega_{8}^{2}, \\
e^{\phi} & =H_{2}^{\frac{3}{4}}, \quad C=\sqrt{1+\alpha^{7}}\left(H_{2} H_{3}\right)^{-\frac{1}{2}} d t,
\end{aligned}
$$

where

$$
H_{i}=1+\frac{r_{-}^{7}}{r^{7}}+\frac{\gamma}{r_{-}^{6} \alpha^{13}} h_{i}\left(\frac{r}{r_{-} \alpha}\right), \quad F_{1}=1-\frac{r_{-}^{7} \alpha^{7}}{r^{7}}+\frac{\gamma}{r_{-}^{6} \alpha^{6}} f_{1}\left(\frac{r}{r_{-} \alpha}\right) .
$$


And the functions $h_{i}(x)$ and $f_{1}(x)$ are uniquely determined as

$$
\begin{aligned}
h_{1}(x)= & \frac{1302501760}{9 x^{34}}-\frac{224\left(2308761-4623760 \alpha^{7}\right)}{9 x^{27}}+\frac{1721664\left(7+13 \alpha^{7}\right)}{13 x^{20}} \\
& -\frac{956480\left(5-24 \alpha^{7}\right)}{13 x^{13}}-\frac{3747840}{x^{7}}+\frac{273280\left(15+7 \alpha^{7}\right)}{x^{6}} \\
& -1639680\left(1+\alpha^{7}\right) \frac{x-1}{x^{7}-1}+117120\left(18-\frac{23}{x^{7}}+2 \alpha^{7}\right) I(x), \\
h_{2}(x)= & \frac{19160960}{x^{34}}-\frac{224\left(2351583+421120 \alpha^{7}\right)}{9 x^{27}}+\frac{81984\left(27+104 \alpha^{7}\right)}{13 x^{20}} \\
& -\frac{409920\left(3-32 \alpha^{7}\right)}{13 x^{13}}-\frac{2108160}{x^{7}}+\frac{273280\left(9+8 \alpha^{7}\right)}{x^{6}} \\
& +1054080\left(2-\frac{1}{x^{7}}+\frac{16}{9} \alpha^{7}\right) I(x), \\
h_{3}(x)= & \frac{361110400}{9 x^{34}}-\frac{224\left(2404287-421120 \alpha^{7}\right)}{9 x^{27}}-\frac{81984\left(293+104 \alpha^{7}\right)}{13 x^{20}} \\
& -\frac{136640\left(425+96 \alpha^{7}\right)}{13 x^{13}}-\frac{2108160}{x^{7}}+\frac{273280\left(9-8 \alpha^{7}\right)}{x^{6}} \\
& +117120\left(18-\frac{41}{x^{7}}-16 \alpha^{7}\right) I(x), \\
f_{1}(x)= & -\frac{1208170880}{9 x^{34}}+\frac{161405664}{x^{27}}+\frac{5738880}{13 x^{20}}+\frac{956480}{x^{13}}+\frac{3747840}{x^{7}}+\frac{819840}{x^{7}} I(x),
\end{aligned}
$$

where the function $I(x)$ is defined by the eq. (3.17). Note that $h_{2}(x)+h_{3}(x)$ and $f_{1}(x)$ do not depend on $\alpha$. Below we examine 3 limits in table 1 .

\subsection{The extremal limit}

In the extremal case, the classical solution is described by the eq. (2.2) with $r_{+}=r_{-}$. This is a half BPS solution and does not receive any higher curvature corrections [44]. This is explicitly checked by taking the extremal limit $\alpha \rightarrow 0$ to the solution (4.1) with (4.2) and (4.3). In fact, because of the asymptotic behaviors (3.37), all quantum corrections are irrelevant and the solution is given by the eq. (4.1) with

$$
H_{i}=1+\frac{r_{-}^{7}}{r^{7}}, \quad F_{1}=1
$$

Thus the extremal solution coincides with a half BPS solution and is not affected by the leading quantum correction at 1-loop.

\subsection{The Schwarzschild limit}

The Schwarzschild limit is given by $r_{-} \rightarrow 0$ with $r_{-} \alpha$ fixed. The solution is given by the eq. (4.1) with

$$
H_{i}=1+\frac{\gamma}{r_{-}^{6} \alpha^{6}} \hat{h}_{i}\left(\frac{r}{r_{-} \alpha}\right), \quad F_{1}=1-\frac{r_{-}^{7} \alpha^{7}}{r^{7}}+\frac{\gamma}{r_{-}^{6} \alpha^{6}} f_{1}\left(\frac{r}{r_{-} \alpha}\right)
$$


and

$$
\begin{aligned}
& \hat{h}_{1}(x)=\frac{1035722240}{9 x^{27}}+\frac{1721664}{x^{20}}+\frac{22955520}{13 x^{13}}+\frac{1912960}{x^{6}}-1639680 \frac{x-1}{x^{7}-1}+234240 I(x), \\
& \hat{h}_{2}(x)=-\hat{h}_{3}(x)=-\frac{94330880}{9 x^{27}}+\frac{655872}{x^{20}}+\frac{13117440}{13 x^{13}}+\frac{2186240}{x^{6}}+1873920 I(x) .
\end{aligned}
$$

Notice that $\hat{h}_{2}(x)+\hat{h}_{3}(x)=0$, which means that R-R 1-form gauge field is trivial and the $\mathrm{R}$ - $\mathrm{R}$ charge is zero.

\subsection{The near horizon limit}

If we take the near horizon limit $\alpha \rightarrow 0$ with $\frac{r}{r_{-} \alpha}$ and $\frac{\gamma}{r_{-}^{6} \alpha^{6}}$ fixed, terms of higher powers of $\alpha$ in the eq. (4.3) vanish and the solution is given by the eq. (4.1) with

$$
H_{i}=\frac{r_{-}^{7}}{r^{7}}+\frac{\gamma}{r_{-}^{6} \alpha^{13}} \tilde{h}_{i}\left(\frac{r}{r_{-} \alpha}\right), \quad F_{1}=1-\frac{r_{-}^{7} \alpha^{7}}{r^{7}}+\frac{\gamma}{r_{-}^{6} \alpha^{6}} f_{1}\left(\frac{r}{r_{-} \alpha}\right),
$$

and

$$
\begin{aligned}
\tilde{h}_{1}(x)= & \frac{1302501760}{9 x^{34}}-\frac{57462496}{x^{27}}+\frac{12051648}{13 x^{20}}-\frac{4782400}{13 x^{13}}-\frac{3747840}{x^{7}}+\frac{4099200}{x^{6}} \\
& -1639680 \frac{x-1}{x^{7}-1}+117120\left(18-\frac{23}{x^{7}}\right) I(x), \\
\tilde{h}_{2}(x)= & \frac{19160960}{x^{34}}-\frac{58528288}{x^{27}}+\frac{2213568}{13 x^{20}}-\frac{1229760}{13 x^{13}}-\frac{2108160}{x^{7}}+\frac{2459520}{x^{6}} \\
& +1054080\left(2-\frac{1}{x^{7}}\right) I(x), \\
\tilde{h}_{3}(x)= & \frac{361110400}{9 x^{34}}-\frac{59840032}{x^{27}}-\frac{24021312}{13 x^{20}}-\frac{58072000}{13 x^{13}}-\frac{2108160}{x^{7}}+\frac{2459520}{x^{6}} \\
& +117120\left(18-\frac{41}{x^{7}}\right) I(x) .
\end{aligned}
$$

This is the quantum near horizon geometry of the black 0-brane which is first derived in ref. [29]. Notice that compared with ref. [29] there is an additional $\frac{3747840}{x^{7}}$ term in $f_{1}(x)$. As is clear form the discussions so far, this term is necessary to be consistent with the extremal limit and the Schwarzschild one, which was not obvious in ref. [29]. Anyway, this additional term does not affect the entropy of the black 0-brane since it depends on $f_{1}(x)$ through $7 f_{1}(1)+f_{1}^{\prime}(1)$.

\section{Mass, R-R charge and internal energy of the quantum black 0-brane}

In this section we evaluate the mass, the R-R charge and the internal energy of the quantum black 0-brane. Formulae for the mass and the R-R charge including higher derivative corrections are discussed in the appendix. As a result, we can employ usual ADM mass and charge formulae. 


\subsection{The mass}

The mass of the quantum black 0-brane is calculated by using ADM mass formula. In order to use the formula the metric should be written in Einstein frame. The metric in the Einstein frame $g_{\mu \nu}^{E}$ is written as $g_{\mu \nu}^{E}=e^{-\frac{\phi}{2}} g_{\mu \nu}$, so the line element in the Einstein frame is given by

$$
\begin{aligned}
d s_{E}^{2} & =-H_{1}^{-1} H_{2}^{\frac{1}{8}} F_{1} d t^{2}+H_{2}^{\frac{1}{8}} F_{1}^{-1} d r^{2}+H_{2}^{\frac{1}{8}} r^{2} d \Omega_{8}^{2} \\
& =-H_{1}^{-1} H_{2}^{\frac{1}{8}} F_{1} d t^{2}+H_{2}^{\frac{1}{8}}\left\{\delta_{i j}+\left(F_{1}^{-1}-1\right) \frac{x_{i} x_{j}}{r^{2}}\right\} d x^{i} d x^{j},
\end{aligned}
$$

where $r^{2}=\left(x^{1}\right)^{2}+\cdots+\left(x^{9}\right)^{2}$ and $i, j=1, \cdots, 9$. Then, by using the eq. (3.37), the deviation from the flat space-time is given by

$$
\begin{aligned}
h_{i j} & =H_{2}^{\frac{1}{8}}\left\{\delta_{i j}+\left(F_{1}^{-1}-1\right) \frac{x_{i} x_{j}}{r^{2}}\right\}-\delta_{i j} \\
& =\frac{r_{-}^{7}+1873920 \gamma r_{-} \alpha}{8 r^{7}} \delta_{i j}+\frac{r_{-}^{7} \alpha^{7}-3747840 \gamma r_{-} \alpha}{r^{7}} \frac{x_{i} x_{j}}{r^{2}}+\mathcal{O}\left(\frac{1}{r^{14}}\right) .
\end{aligned}
$$

Now we are ready to apply the ADM mass formula. The mass of the quantum black 0-brane is evaluated as

$$
\begin{aligned}
M & =\frac{1}{2 \kappa_{10}^{2}} \int_{r=\infty} d \Omega_{8} r^{8}\left(\partial_{j} h^{i j}-\partial^{i} h^{j}{ }_{j}\right) \frac{x_{i}}{r} \\
& =\frac{V_{S^{8}}}{2 \kappa_{10}^{2}}\left\{8 r_{-}^{7} \alpha^{7}+7 r_{-}^{7}-16865280 \gamma r_{-} \alpha\right\} .
\end{aligned}
$$

Thus the mass receives the nontrivial quantum correction at 1-loop level.

\subsection{The R-R charge}

The black 0-brane couples to the R-R 1-form field and carries the R-R charge. From the eq. (4.1), the R-R 1-form field $C$ and 2-form field strength $G_{2}=d C$ are given by

$$
\begin{aligned}
C & =\left(\frac{r_{+}}{r_{-}}\right)^{\frac{7}{2}} H_{2}^{-\frac{1}{2}} H_{3}^{-\frac{1}{2}} d t \\
G_{2} & =\frac{1}{2}\left(\frac{r_{+}}{r_{-}}\right)^{\frac{7}{2}} H_{2}^{-\frac{1}{2}} H_{3}^{-\frac{1}{2}}\left(H_{2}^{-1} \frac{d H_{2}}{d r}+H_{3}^{-1} \frac{d H_{3}}{d r}\right) d t \wedge d r .
\end{aligned}
$$

Then the R-R charge of the quantum black 0-brane is evaluated as

$$
\begin{aligned}
Q & =\frac{1}{2 \kappa_{10}^{2}} \int_{r=\infty} * G_{2} \\
& =\frac{1}{2 \kappa_{10}^{2}} \int_{r=\infty} d \Omega_{8}\left\{-\frac{1}{2}\left(\frac{r_{+}}{r_{-}}\right)^{\frac{7}{2}} H_{1}^{\frac{1}{2}} H_{2} H_{3}^{-\frac{1}{2}}\left(H_{2}^{-1} \frac{d H_{2}}{d r}+H_{3}^{-1} \frac{d H_{3}}{d r}\right) r^{8}\right\} \\
& =\frac{1}{2 \kappa_{10}^{2}} \int_{r=\infty} d \Omega_{8}\left(\frac{r_{+}}{r_{-}}\right)^{\frac{7}{2}}\left[-r^{8} \frac{d H}{d r}-\frac{\gamma r^{8}}{2 r_{-}^{6} \alpha^{13}} \frac{d}{d r}\left\{h_{2}\left(\frac{r}{r_{-} \alpha}\right)+h_{3}\left(\frac{r}{r_{-} \alpha}\right)\right\}\right] \\
& =\frac{V_{S^{8}}}{2 \kappa_{10}^{2}} 7 r_{-}^{7} \sqrt{1+\alpha^{7}} .
\end{aligned}
$$

Quantum corrections do not contribute to the R-R charge because $h_{2}\left(\frac{r}{r_{-} \alpha}\right)+h_{3}\left(\frac{r}{r_{-} \alpha}\right) \sim \frac{1}{r^{14}}$ when $r \rightarrow \infty$. Therefore the $\mathrm{R}-\mathrm{R}$ charge remains the same as the classical one. 


\section{Validity of the quantum black 0-brane solution}

So far we constructed the quantum black 0-brane solution by considering the effective action (3.1). After the dimensional reduction, this corresponds to the leading quantum correction to the type IIA supergravity. However, this is a part of the effective action in the type IIA superstring theory, so the black 0-brane is affected by other higher derivative corrections in general. In this section, we examine those corrections and clarify the validity of the solution (4.1).

Since we compactify the 11 dimensional direction on the circle, when the radius of the circle is finite, the Kaluza-Klein modes give nontrivial contributions to the effective action. This corresponds to the leading tree level effective action, which is expressed by by $e^{-2 \phi} R^{4}$ [45]. It is also known that there are terms of $e^{2 \phi} \partial^{4} R^{4}$ at two loop level [46]. Although the full structure of the effective action of the type IIA superstring theory is not completed yet, from the string duality arguments, it takes the following form [47]

$$
\begin{aligned}
g_{s}^{2} e^{2 \phi} \mathcal{L} \sim R & +\left(\alpha^{\prime 3} R^{4}+\alpha^{\prime 5} \partial^{4} R^{4}+\cdots\right)+g_{s}^{2} e^{2 \phi}\left(\alpha^{\prime 3} R^{4}+\alpha^{\prime 6} \partial^{6} R^{4}+\cdots\right) \\
& +\left(g_{s}^{2} e^{2 \phi}\right)^{2}\left(\alpha^{\prime 5} \partial^{4} R^{4}+\cdots\right)+\cdots+\left(g_{s}^{2} e^{2 \phi}\right)^{n}\left(\alpha^{\prime 3+n} \partial^{2 n} R^{4}+\cdots\right)+\cdots,
\end{aligned}
$$

where $\alpha^{\prime}=\ell_{s}^{2}$. The Riemann tensor behaves like $R_{a b c d} \sim E^{2}$, where $E$ is a typical energy of the black 0-brane, such as the internal energy. Then the dimensional analysis shows that

$$
\begin{aligned}
g_{s}^{2} e^{2 \phi} \mathcal{L} \sim E^{2}\{1 & +\left(\alpha^{\prime 3} E^{6}+\alpha^{\prime 5} E^{10}+\cdots\right)+g_{s}^{2}\left(\alpha^{\prime 3} E^{6}+\alpha^{\prime 6} E^{12}+\cdots\right) \\
& \left.+g_{s}^{4}\left(\alpha^{\prime 5} E^{10}+\cdots\right)+\cdots+g_{s}^{2 n}\left(\alpha^{\prime 3+n} E^{6+2 n}+\cdots\right)+\cdots\right\} .
\end{aligned}
$$

The leading 1-loop quantum correction $g_{s}^{2} \alpha^{\prime 3} E^{6}$ becomes sub-dominant when

$$
1<g_{s}^{2}<\frac{1}{\alpha^{\prime} E^{2}}
$$

The solution (4.1) is valid in this region. This means that the radius of the 11 th direction $g_{s} \ell_{s}$ is large compared to the string scale, and the typical energy $E$ is small compared to the Kaluza-Klein mass $\frac{1}{g_{s} \ell_{s}}$.

\section{Conclusion and discussion}

In this paper we investigated the quantum corrections to the non-extremal M-wave and black 0-brane solutions. The effective action for the M-theory is taken into account including higher curvature $R^{4}$ terms, and the equations of motion for the non-extremal M-wave are analytically solved up to the leading quantum level. The Kaluza-Klein reduction of the quantum M-wave gives the quantum black 0-brane solution which is asymptotically flat. The explicit form of the solution is given by eqs. (4.1)-(4.3). The integral constants of the solution are uniquely fixed by imposing asymptotic flatness and finiteness around the event horizon. We also required consistencies with the extremal limit and the Schwarzschild one in the table 1. The extremal limit of the solution remains the same as the classical one, 
so it is not affected by the leading quantum corrections. The Schwarzschild limit of the quantum black 0-brane is given by eqs. (4.5) and (4.6). The near horizon limit is done by eqs. (4.7) and (4.8). Notice that the near horizon limit of the metric is the same as that in ref. [29] except $\frac{1}{x^{7}}$ term in $f_{1}(x)$. This extra term was dropped in ref. [29] by imposing stronger boundary condition. Nevertheless, this does not affect physical quantities, such as the near horizon limit of the internal energy.

The quantum corrections to the ADM mass and the R-R charge are discussed in the appendix. We employed Noether and Wald's method with the vielbein formalism. After some calculations we concluded that the corrections are suppressed at the spacial infinity and the ADM mass and the charge formulae are still valid for the quantum black 0-brane. The mass of the quantum black 0-brane receives the quantum correction, and the R-R charge remains the same as the classical one. This would be a renormalization of the mass of the gravitational objects.

One of the important future works is to generalize the above discussions to the other black $p$-branes. Although our knowledge of the effective action for the superstring theory is limited, it is possible to consider quantum corrections to the black 6-brane, since this is uplifted to a Kaluza-Klein monopole solution in 11 dimensions. It is also possible to construct Schwarzschild black hole solutions in various dimensions by compactifications. Schwarzschild black hole in 11 dimensions is important from the viewpoint of the test of gauge gravity duality. The solution in 4 dimensions will also be important because it could give some astrophysical predictions to be observed in the future. For other black $p$-branes, first we need to know the effective action including $\mathrm{R}-\mathrm{R}$ gauge fields.

\section{Acknowledgments}

The author would like to thank Masanori Hanada, Goro Ishiki, Hikaru Kawai and Jun Nishimura for the motivation of this work. He also got some insights from many colleagues through discussions on ref. [36]. This work was partially supported by the Ministry of Education, Science, Sports and Culture, Grant-in-Aid for Young Scientists (B) 24740140, 2012.

\section{A Higher derivative corrections to the ADM mass and the R-R charge}

Since the effective action of the M-theory (3.1) contains higher curvature terms, the ADM mass formula and the charge of the non-extremal M-wave or the black 0-brane would be affected by these terms. In this section we derive higher curvature corrections to the ADM mass and the charge via Noether and Wald's method [48, 49]. We use the vielbein formalism which is important for the supergravity [50].

First, the variation of the Lagrangian (3.1) is evaluated as

$$
\begin{aligned}
2 \kappa_{11}^{2} \delta \mathcal{L}= & e\left(\eta^{a c} \eta^{b d}+\gamma X^{a b c d}\right) \delta R_{a b c d}-e e^{a}{ }_{M}\left\{R+\gamma\left(t_{8} t_{8} R^{4}-\frac{1}{4 !} \epsilon_{11} \epsilon_{11} R^{4}\right)\right\} \delta e^{M}{ }_{a} \\
= & 2 e\left[R_{i j}-\frac{1}{2} \eta_{i j} R+\gamma\left\{R_{a b c i} X^{a b c}{ }_{j}-\frac{1}{2} \eta_{i j}\left(t_{8} t_{8} R^{4}-\frac{1}{4 !} \epsilon_{11} \epsilon_{11} R^{4}\right)\right\}\right] \delta e^{i j} \\
& -2 e\left(\eta^{a c} \eta^{b d}+\gamma X^{a b c d}\right) D_{d} \delta \omega_{c a b}
\end{aligned}
$$




$$
\begin{aligned}
= & 2 e\left[R_{i j}-\frac{1}{2} \eta_{i j} R+\gamma\left\{R_{a b c i} X_{j}^{a b c}-\frac{1}{2} \eta_{i j}\left(t_{8} t_{8} R^{4}-\frac{1}{4 !} \epsilon_{11} \epsilon_{11} R^{4}\right)\right\}\right] \delta e^{i j} \\
& +2 \gamma e D_{d} X^{a b c d}\left(\delta_{a}^{k} \eta_{b i} \eta_{c j}+\delta_{a}^{k} \eta_{b j} \eta_{c i}+\delta_{c}^{k} \eta_{i a} \eta_{b j}\right) D_{k} \delta e^{i j} \\
& -2 \partial_{M}\left\{e\left(e^{N a} e^{M b}+\gamma X^{a b N M}\right) \delta \omega_{N a b}\right\} \\
= & 2 e E_{i j} \delta e^{i j}++\partial_{M}\left(e \Theta^{M}(\delta)\right),
\end{aligned}
$$

where $\delta e^{i j}=e^{i}{ }_{M} \delta e^{M j}$ and $\Theta^{M}(\delta)$ is defined by

$$
\Theta^{M}(\delta)=2 e^{M a} e^{N b} \delta \omega_{N a b}+2 \gamma\left\{X^{a b M N} \delta \omega_{N a b}+e^{M a}\left(D^{b} X_{a i j b}+D^{b} X_{a j i b}+D^{b} X_{a b i j}\right) \delta e^{i j}\right\} .
$$

$E_{i j}=0$ is the equations of motion given by the eq. (3.3). The variation of the Lagrangian becomes total derivative term up to $E_{i j}=0$. Since these variations are not covariant under the local Lorentz transformation, we consider following field dependent local Lorentz transformation simultaneously.

$$
\begin{aligned}
\delta_{L} e^{M}{ }_{a} & =-e^{M}{ }_{b} \epsilon^{b}, \\
\delta_{L} \omega_{N}{ }^{a b} & =-D_{N} \epsilon^{a b}, \\
\epsilon^{a b} & =\frac{1}{2} e^{a}{ }_{P} \delta e^{P b}-\frac{1}{2} e^{b}{ }_{P} \delta e^{P a} .
\end{aligned}
$$

Then the variations of fields with (A.3) becomes

$$
\begin{aligned}
\bar{\delta} e^{M}{ }_{a} & =-\frac{1}{2} g^{M N} \delta g_{N P} e_{a}^{P}, \\
\bar{\delta} \omega_{N}^{a b} & =\frac{1}{2} e^{a P} e^{b Q}\left(\nabla_{Q} \delta g_{P N}-\nabla_{P} \delta g_{Q N}\right),
\end{aligned}
$$

where $\nabla_{P}$ represents a covariant derivative with respect to space-time indices. These variations are covariant under local Lorentz transformation. In the following we write $\bar{\delta}$ as $\delta$ for simplicity. Now we consider the variation for the general coordinate transformation $x^{M}=x^{M}-\xi^{M}$. In this case the variation (A.4) becomes

$$
\begin{aligned}
\delta_{\xi} e^{M}{ }_{a} & =-\frac{1}{2} e^{M b}\left(D_{a} \xi_{b}+D_{b} \xi_{a}\right), \\
\delta_{\xi} \omega_{N}{ }^{a b} & =\frac{1}{2} e_{c N}\left\{D^{b}\left(D^{c} \xi^{a}+D^{a} \xi^{c}\right)-D^{a}\left(D^{c} \xi^{b}+D^{b} \xi^{c}\right)\right\} \\
& =\xi^{P} R^{a b}{ }_{P N}-\frac{1}{2} D_{N}\left(D^{a} \xi^{b}-D^{b} \xi^{a}\right) .
\end{aligned}
$$

Inserting the eq. (A.5) into the eq. (A.2), $\Theta^{M}(\xi)=\Theta^{M}\left(\delta_{\xi}\right)$ is evaluated as

$$
\begin{aligned}
e \Theta^{M}(\xi)= & 2 e R^{M}{ }_{N} \xi^{N}-e e^{M}{ }_{a} e^{N}{ }_{b} D_{N}\left(D^{a} \xi^{b}-D^{b} \xi^{a}\right) \\
& +2 \gamma\left\{e R_{a b c i} X^{a b c M} \xi^{i}-e e^{M}{ }_{j} X^{a j i b} D_{a} D_{b} \xi_{i}+e e^{M}{ }_{j}\left(D_{b} X^{i j a b}+D_{b} X^{a j i b}\right) D_{a} \xi_{i}\right\} \\
= & 2 e R^{M}{ }_{N} \xi^{N}+2 \gamma\left\{e R_{a b c i} X^{a b c M} \xi^{i}-e e^{M}{ }_{j}\left(D_{a} D_{b} X^{i j a b}+2 D_{(a} D_{b)} X^{a i j b}\right) \xi_{i}\right\} \\
& +\partial_{N}\left[-2 e \nabla^{[M} \xi^{N]}-2 \gamma e\left\{X^{M N P Q} \nabla_{P} \xi_{Q}+e^{M}{ }_{i} e^{N}{ }_{a}\left(D_{b} X^{i j a b}-2 D_{b} X^{a(i j) b}\right) \xi_{j}\right\}\right] \\
= & \partial_{N}\left[-2 e \nabla^{[M} \xi^{N]}-2 \gamma e\left\{X^{M N P Q} \nabla_{P} \xi_{Q}+e^{M}{ }_{i} e^{N}{ }_{a}\left(D_{b} X^{i j a b}-2 D_{b} X^{a(i j) b}\right) \xi_{j}\right\}\right] \\
& +2 \kappa_{11}^{2} \xi^{M} \mathcal{L}+2 e e^{M j} \xi^{i} E_{i j} .
\end{aligned}
$$


Therefore the variation of the Lagrangian is given by

$$
\begin{aligned}
2 \kappa_{11}^{2} \delta_{\xi} \mathcal{L} & =\partial_{M}\left(e \Theta^{M}(\xi)\right)+2 e E_{i j} \delta e^{i j} \\
e \Theta^{M}(\xi) & =\partial_{N}\left(e Q^{M N}(\xi)\right)+2 \kappa_{11}^{2} \xi^{M} \mathcal{L}+2 e e^{M j} \xi^{i} E_{i j}, \\
e Q^{M N}(\xi) & =-2 e \nabla^{[M} \xi^{N]}-2 \gamma e\left\{X^{M N P Q} \nabla_{P} \xi_{Q}+e^{M}{ }_{i} e^{N}{ }_{a}\left(D_{b} X^{i j a b}-2 D_{b} X^{a(i j) b}\right) \xi_{j}\right\}
\end{aligned}
$$

Next, let us consider the variation of the Lagrangian by using the general covariance. Since $e^{-1} \mathcal{L}$ transforms as a scalar field, the Lagrangian does like

$$
\delta_{\xi} \mathcal{L}=\partial_{M}\left(\xi^{M} \mathcal{L}\right)
$$

From the eqs. (A.7) and (A.8), the Noether current is constructed as

$$
\begin{aligned}
2 \kappa_{11}^{2} e J^{M}(\xi) & =e \Theta^{M}(\xi)-2 \kappa_{11}^{2} \xi^{M} \mathcal{L}+\partial_{N}\left(e \tilde{Q}^{M N}(\xi)\right) \\
& =\partial_{N}\left\{e\left(Q^{M N}(\xi)+\tilde{Q}^{M N}(\xi)\right)\right\}+2 e e^{M j} \xi^{i} E_{i j} .
\end{aligned}
$$

Here $\tilde{Q}^{M N}(\xi)$ is an antisymmetric tensor and represents the ambiguity of the current. In order to fix $\tilde{Q}^{M N}(\xi)$, let us consider the variation of the current.

$$
\begin{aligned}
\delta\left(2 \kappa_{11}^{2} e J^{M}(\xi)\right) & =\delta\left(e \Theta^{M}(\xi)\right)-2 \kappa_{11}^{2} \xi^{M} \delta \mathcal{L}+\partial_{N}\left\{\delta\left(e \tilde{Q}^{M N}(\xi)\right)\right\} \\
& =\delta\left(e \Theta^{M}(\xi)\right)-\xi^{M} \partial_{N}\left(e \Theta^{N}(\delta)\right)-2 e \xi^{M} E_{i j} \delta e^{i j}+\partial_{N}\left\{\delta\left(e \tilde{Q}^{M N}(\xi)\right)\right\} \\
& =e \omega^{M}(\xi, \delta)+\partial_{N}\left\{-2 e \xi^{[M} \Theta^{N]}(\delta)+\delta\left(e \tilde{Q}^{M N}(\xi)\right)\right\}-2 e \xi^{M} E_{i j} \delta e^{i j},
\end{aligned}
$$

where we defined the symplectic current

$$
e \omega^{M}(\xi, \delta)=\delta\left(e \Theta^{M}(\xi)\right)-\delta_{\xi}^{\mathrm{L}}\left(e \Theta^{M}(\delta)\right) .
$$

The integral of the symplectic current over Cauchy surface gives the variation of the Hamiltonian. Therefore in order to obtain correct equations of motion, we should eliminate the surface term in the eq. (A.10). So we choose the variation of $\tilde{Q}^{M N}(\xi)$ as

$$
\delta\left(e \tilde{Q}^{M N}(\xi)\right)=2 e \xi^{[M} \Theta^{N]}(\delta) .
$$

From the eq. (A.9), the variation of the current is given by

$$
\delta\left(2 \kappa_{11}^{2} e J^{M}(\xi)\right)=\partial_{N}\left\{\delta\left(e Q^{M N}(\xi)\right)+2 e \xi^{[M} \Theta^{N]}(\delta)\right\}+\delta\left(2 e e^{M j} \xi^{i} E_{i j}\right) .
$$

Conserved quantities are obtained by integrating $e j^{t}(\xi)$ over 10 dimensional space.

When $\xi$ is chosen as an asymptotic time translation $\xi_{T}$ and the variation of the fields, such as $\delta e^{M}$, satisfy linearized equations of motion, the variation of the mass is given by

$$
\begin{aligned}
\delta M= & \frac{1}{2 \kappa_{11}^{2}} \int_{r=\infty}\left\{\delta\left(e Q^{\operatorname{tr}}\left(\xi_{T}\right)\right)+2 e \xi_{T}^{[t} \Theta^{r]}(\delta)\right\} \\
= & \frac{1}{2 \kappa_{11}^{2}} \int_{r=\infty}\left\{\delta\left(-2 e \nabla^{[t} \xi_{T}^{r]}\right)+4 e \xi_{T}^{[t} e^{r] a} e^{N b} \delta \omega_{N a b}\right\} \\
& +\frac{\gamma}{2 \kappa_{11}^{2}} \int_{r=\infty}\left[\delta\left\{-2 e X^{\operatorname{tr} P Q} \nabla_{P} \xi_{T Q}-2 e e_{i}^{t} e^{r}{ }_{a}\left(D_{b} X^{i j a b}-2 D_{b} X^{a(i j) b}\right) \xi_{T j}\right\}\right.
\end{aligned}
$$




$$
\begin{array}{r}
\left.+4 e \xi_{T}^{[t} X^{r] N a b} \delta \omega_{N a b}+4 e \xi_{T}^{[t} e^{r] a}\left(D^{b} X_{a i j b}+D^{b} X_{a j i b}+D^{b} X_{a b i j}\right) \delta e^{i j}\right] \\
=\frac{1}{2 \kappa_{11}^{2}} \int_{r=\infty}\left\{\delta\left(-2 e \nabla^{[t} \xi_{T}^{r]}\right)+2 e \xi_{T}^{[t} g^{r] P} g^{N Q}\left(\nabla_{Q} \delta g_{P N}-\nabla_{P} \delta g_{Q N}\right)\right\} .
\end{array}
$$

Since each non zero component of the Riemann tensor behaves like $R_{a b c d} \sim \mathcal{O}\left(\frac{1}{r^{9}}\right)$ asymptotically, in the above we dropped terms which depend on $X_{a b c d}$. The last equation is equivalent to the variation of the ADM mass [49]. Therefore the mass is simply given by the ADM mass formula.

When $\xi$ is chosen as an asymptotic translation along $z$ direction $\xi_{Z}$, it satisfies $\xi_{Z}^{t} \Theta^{r}(\delta)-\xi_{Z}^{r} \Theta^{t}(\delta)=0$, and the charge is given by

$$
\begin{aligned}
Q= & \frac{1}{2 \kappa_{11}^{2}} \int_{r=\infty} e Q^{t r}\left(\xi_{Z}\right) \\
= & \frac{1}{2 \kappa_{11}^{2}} \int_{r=\infty}\left(-2 e \nabla^{[t} \xi_{Z}^{r]}\right) \\
& +\frac{\gamma}{2 \kappa_{11}^{2}} \int_{r=\infty}\left\{-2 e X^{t r P Q} \nabla_{P} \xi_{Z Q}-2 e e_{i}^{t} e^{r}{ }_{a}\left(D_{b} X^{i j a b}-2 D_{b} X^{a(i j) b}\right) \xi_{Z j}\right\} \\
= & \frac{1}{2 \kappa_{10}^{2}} \int_{r=\infty} \sqrt{-g} G^{t r} .
\end{aligned}
$$

As explained in the mass formula, terms which depend on $X_{a b c d}$ are dropped, and the dimensional reduction (2.3) is used in the last step. Thus the R-R charge is given by the integral of the R-R flux as usual.

Finally we choose the Killing vector as $\xi=\xi_{T}+\Omega \xi_{Z}$, which becomes zero at the bifurcate horizon $\Sigma$. Then $\delta_{\xi} e^{M}{ }_{a}=\delta_{\xi} \omega_{N}{ }^{a b}=0$, and the symplectic current and the variation of the current also vanish. Furthermore, if the variation of the fields, such as $\delta e^{M}{ }_{a}$, satisfy linearized equations of motion, the eq. (A.13) is simplified as

$$
0=\frac{1}{2 \kappa_{11}^{2}} \partial_{N}\left\{\delta\left(e Q^{M N}(\xi)\right)+e\left(\xi^{M} \Theta^{N}(\delta)-\xi^{N} \Theta^{M}(\delta)\right)\right\}
$$

By integrating the above equation over 10 dimensional asymptotically flat space with the horizon, we derive the first law of the black hole,

$$
\delta M+\Omega \delta Q=\frac{\kappa}{2 \pi} \delta S .
$$

Here $\kappa$ is the surface gravity which is given by $\nabla_{M} \xi_{N}=\kappa N_{M N}$ at the bifurcate horizon $\Sigma$, and $N_{M N}$ is an antisymmetric tensor which is binormal to the bifurcate horizon $\Sigma$. $S$ corresponds to the entropy and is given by

$$
S=-\frac{2 \pi}{2 \kappa_{11}^{2}} \int_{Q} \sqrt{h}\left(g^{M P} g^{N Q}+\gamma X^{M N P Q}\right) N_{M N} N_{P Q}
$$

$\sqrt{h}$ is a volume factor of $\Sigma$. 


\section{B Near horizon limit of the internal energy}

The internal energy $E$ of the quantum black 0-brane is defined by $E=M-Q$. From the eqs. (5.3) and (5.5), the internal energy is written as

$$
E=\frac{V_{S^{8}}}{2 \kappa_{10}^{2}}\left(8 r_{-}^{7} \alpha^{7}+7 r_{-}^{7}-7 r_{-}^{7} \sqrt{1+\alpha^{7}}-16865280 \gamma r_{-} \alpha\right) .
$$

Below we consider the near horizon limit of the internal energy, which was first derived in ref. [29] by using the black hole thermodynamics.

Since we will express $E$ in terms of the temperature in the near horizon limit, first we need to derive the relation between parameters in the solution and the temperature. The location of the event horizon $r=r_{H}$ is obtained by solving $F_{1}\left(r_{H}\right)=0$ and modified up to the linear order of $\gamma$ as

$$
r_{H}=r_{-} \alpha-\frac{\gamma}{7 r_{-}^{5} \alpha^{5}} f_{1}(1)
$$

The Hawking temperature of the black 0-brane is given by

$$
\begin{aligned}
T & =\left.\frac{1}{4 \pi} H_{1}^{-\frac{1}{2}} \frac{d F_{1}}{d r}\right|_{r_{H}} \\
& =\frac{7 r_{-}^{\frac{5}{2}} \alpha^{\frac{5}{2}}}{4 \pi r_{-}^{\frac{7}{2}} \sqrt{1+\alpha^{7}}}\left[1+\frac{\gamma}{r_{-}^{6} \alpha^{6}}\left\{\left(\frac{8}{7}-\frac{1}{2\left(1+\alpha^{7}\right)}\right) f_{1}(1)+\frac{1}{7} f_{1}^{\prime}(1)-\frac{1}{2\left(1+\alpha^{7}\right)} h_{1}(1)\right\}\right]
\end{aligned}
$$

up to the linear order of $\gamma$. Now let us take the near horizon limit,

$$
\alpha \rightarrow 0, \frac{r_{ \pm}^{7}}{\ell_{s}^{10}} \rightarrow(2 \pi)^{4} 15 \pi \lambda \quad \text { with } \quad U_{0}=\frac{r_{-} \alpha}{\ell_{s}^{2}}, \frac{\gamma}{r_{-}^{6} \alpha^{6}}=\frac{\pi^{6} \lambda^{2}}{2^{7} 3^{2} N^{2} U_{0}^{6}} \quad \text { fixed } .
$$

Then the temperature (B.3) approaches to

$$
\tilde{T}=a_{1} \tilde{U}_{0}^{\frac{5}{2}}\left(1+\epsilon a_{2} \tilde{U}_{0}^{-6}\right),
$$

where

$$
\begin{aligned}
a_{1} & =\frac{7}{16 \pi^{3} \sqrt{15 \pi}} \sim 2.06 \times 10^{-3}, \\
a_{2} & =\frac{9}{14} f_{1}(1)+\frac{1}{7} f_{1}^{\prime}(1)-\frac{1}{2} h_{1}(1) \sim-4.02 \times 10^{5}, \\
\epsilon & =\frac{\pi^{6}}{2^{7} 3^{2} N^{2}} \sim \frac{0.835}{N^{2}} .
\end{aligned}
$$

Here dimensional quantities $\tilde{T} \equiv T / \lambda^{\frac{1}{3}}$ and $\tilde{U}_{0} \equiv U_{0} / \lambda^{\frac{1}{3}}$ are introduced to make expressions simple. From the eq. (B.5), it is possible to express $\tilde{U}_{0}$ in terms of $\tilde{T}$ as

$$
\tilde{U}_{0}=a_{1}^{-\frac{2}{5}} \tilde{T}^{\frac{2}{5}}\left(1-\frac{2}{5} \epsilon a_{1}^{\frac{12}{5}} a_{2} \tilde{T}^{-\frac{12}{5}}\right)
$$

By using this relation, the near horizon limit of the internal energy is expressed as a function of the temperature. 
Finally let us evaluate the near horizon limit of the internal energy. By taking the near horizon limit (B.4) and using $2 \kappa_{10}^{2}=(2 \pi)^{7} \ell_{s}^{8} g_{s}^{2}=\frac{(2 \pi)^{11} \ell_{s}^{14} \lambda^{2}}{N^{2}}, V_{S^{8}}=\frac{2(2 \pi)^{4}}{7 \cdot 15}$ and the eq. (B.7), it is calculated as follows.

$$
\begin{aligned}
E & =\frac{2 N^{2}}{(2 \pi)^{7} 105 \ell_{s}^{14} \lambda^{2}}\left(\frac{9}{2} U_{0}^{7} \ell_{s}^{14}-16865280 \epsilon \lambda^{2} U_{0} \ell_{s}^{14}\right) \\
& =\frac{9 N^{2} \lambda^{\frac{1}{3}}}{(2 \pi)^{7} 105}\left(\tilde{U}_{0}^{7}-3747840 \epsilon \tilde{U}_{0}\right) \\
& =\frac{9 N^{2} \lambda^{\frac{1}{3}}}{(2 \pi)^{7} 105}\left\{a_{1}^{-\frac{14}{5}} \tilde{T}^{\frac{14}{5}}-\epsilon\left(\frac{14}{5} a_{2}+3747840\right) a_{1}^{-\frac{2}{5}} \tilde{T}^{\frac{2}{5}}\right\} .
\end{aligned}
$$

By inserting numerical values of (B.6), the dimensionless internal energy of the quantum black 0-brane $\tilde{E}=E / \lambda^{\frac{1}{3}}$ is written as

$$
\frac{\tilde{E}}{N^{2}}=7.41 \tilde{T}^{\frac{14}{5}}-\frac{5.77}{N^{2}} \tilde{T}^{\frac{2}{5}}
$$

This is exactly the same as the result derived in ref. [29]. The above relation is also reproduced by the numerical simulation from the dual gauge theory [36], which strongly supports the gauge gravity duality at the quantum level.

Open Access. This article is distributed under the terms of the Creative Commons Attribution License (CC-BY 4.0), which permits any use, distribution and reproduction in any medium, provided the original author(s) and source are credited.

\section{References}

[1] J. Polchinski, Dirichlet branes and Ramond-Ramond charges, Phys. Rev. Lett. 75 (1995) 4724 [hep-th/9510017] [INSPIRE].

[2] G.W. Gibbons and K.-I. Maeda, Black holes and membranes in higher dimensional theories with dilaton fields, Nucl. Phys. B 298 (1988) 741 [INSPIRE].

[3] G.T. Horowitz and A. Strominger, Black strings and p-branes, Nucl. Phys. B 360 (1991) 197 [INSPIRE].

[4] A. Strominger and C. Vafa, Microscopic origin of the Bekenstein-Hawking entropy, Phys. Lett. B 379 (1996) 99 [hep-th/9601029] [INSPIRE].

[5] J.M. Maldacena, The large- $N$ limit of superconformal field theories and supergravity, Int. J. Theor. Phys. 38 (1999) 1113 [hep-th/9711200] [INSPIRE].

[6] S.S. Gubser, I.R. Klebanov and A.M. Polyakov, Gauge theory correlators from noncritical string theory, Phys. Lett. B 428 (1998) 105 [hep-th/9802109] [inSPIRE].

[7] E. Witten, Anti-de Sitter space and holography, Adv. Theor. Math. Phys. 2 (1998) 253 [hep-th/9802150] [INSPIRE].

[8] A. Dabholkar, Exact counting of black hole microstates, Phys. Rev. Lett. 94 (2005) 241301 [hep-th/0409148] [INSPIRE].

[9] H. Ooguri, A. Strominger and C. Vafa, Black hole attractors and the topological string, Phys. Rev. D 70 (2004) 106007 [hep-th/0405146] [INSPIRE]. 
[10] D.J. Gross and E. Witten, Superstring modifications of Einstein's equations, Nucl. Phys. B 277 (1986) 1 [INSPIRE].

[11] D.J. Gross and J.H. Sloan, The quartic effective action for the heterotic string, Nucl. Phys. B 291 (1987) 41 [inSPIRE].

[12] M.T. Grisaru, A.E.M. van de Ven and D. Zanon, Four loop $\beta$-function for the $N=1$ and $N=2$ supersymmetric nonlinear $\sigma$-model in two-dimensions, Phys. Lett. B 173 (1986) 423 [INSPIRE].

[13] M.T. Grisaru and D. Zanon, $\sigma$ model superstring corrections to the Einstein-Hilbert action, Phys. Lett. B 177 (1986) 347 [INSPIRE].

[14] A.A. Tseytlin, $R^{4}$ terms in 11 dimensions and conformal anomaly of $(2,0)$ theory, Nucl. Phys. B 584 (2000) 233 [hep-th/0005072] [inSPIRE].

[15] K. Becker and M. Becker, Supersymmetry breaking, M-theory and fluxes, JHEP 07 (2001) 038 [hep-th/0107044] [INSPIRE].

[16] G. Policastro and D. Tsimpis, $R^{4}$, purified, Class. Quant. Grav. 23 (2006) 4753 [hep-th/0603165] [INSPIRE].

[17] M. de Roo, H. Suelmann and A. Wiedemann, Supersymmetric $R^{4}$ actions in ten-dimensions, Phys. Lett. B 280 (1992) 39 [INSPIRE].

[18] M. de Roo, H. Suelmann and A. Wiedemann, The supersymmetric effective action of the heterotic string in ten-dimensions, Nucl. Phys. B 405 (1993) 326 [hep-th/9210099] [INSPIRE].

[19] H. Suelmann, String effective actions and supersymmetry, Ph.D. thesis, Groningen University, Groningen The Netherlands (1994) [INSPIRE].

[20] K. Peeters, P. Vanhove and A. Westerberg, Supersymmetric higher derivative actions in ten-dimensions and eleven-dimensions, the associated superalgebras and their formulation in superspace, Class. Quant. Grav. 18 (2001) 843 [hep-th/0010167] [INSPIRE].

[21] Y. Hyakutake and S. Ogushi, Higher derivative corrections to eleven dimensional supergravity via local supersymmetry, JHEP 02 (2006) 068 [hep-th/0601092] [INSPIRE].

[22] Y. Hyakutake, Toward the determination of $R^{3} F^{2}$ terms in $M$-theory, Prog. Theor. Phys. 118 (2007) 109 [hep-th/0703154] [INSPIRE].

[23] C.G. Callan Jr., R.C. Myers and M.J. Perry, Black holes in string theory, Nucl. Phys. B 311 (1989) 673 [inSPIRE].

[24] S.S. Gubser, I.R. Klebanov and A.A. Tseytlin, Coupling constant dependence in the thermodynamics of $N=4$ supersymmetric Yang-Mills theory, Nucl. Phys. B 534 (1998) 202 [hep-th/9805156] [INSPIRE].

[25] S. de Haro, A. Sinkovics and K. Skenderis, On $\alpha^{\prime}$ corrections to D-brane solutions, Phys. Rev. D 68 (2003) 066001 [hep-th/0302136] [INSPIRE].

[26] A. Sen, Stretching the horizon of a higher dimensional small black hole, JHEP 07 (2005) 073 [hep-th/0505122] [INSPIRE].

[27] A. Buchel, Higher derivative corrections to near-extremal black holes in type IIB supergravity, Nucl. Phys. B 750 (2006) 45 [hep-th/0604167] [INSPIRE].

[28] H. Saida and J. Soda, Statistical entropy of BTZ black hole in higher curvature gravity, Phys. Lett. B 471 (2000) 358 [gr-qc/9909061] [INSPIRE]. 
[29] Y. Hyakutake, Quantum near-horizon geometry of a black 0-brane, Prog. Theor. Exp. Phys. 2014 (2014) 033B04 [arXiv:1311.7526] [INSPIRE].

[30] D.N. Kabat, G. Lifschytz and D.A. Lowe, Black hole thermodynamics from calculations in strongly coupled gauge theory, Int. J. Mod. Phys. A 16 (2001) 856 [hep-th/0007051] [INSPIRE].

[31] M. Hanada, J. Nishimura and S. Takeuchi, Non-lattice simulation for supersymmetric gauge theories in one dimension, Phys. Rev. Lett. 99 (2007) 161602 [arXiv:0706.1647] [INSPIRE].

[32] S. Catterall and T. Wiseman, Towards lattice simulation of the gauge theory duals to black holes and hot strings, JHEP 12 (2007) 104 [arXiv:0706.3518] [INSPIRE].

[33] M. Hanada, Y. Hyakutake, J. Nishimura and S. Takeuchi, Higher derivative corrections to black hole thermodynamics from supersymmetric matrix quantum mechanics, Phys. Rev. Lett. 102 (2009) 191602 [arXiv:0811.3102] [inSPIRE].

[34] D. Kadoh and S. Kamata, One dimensional supersymmetric Yang-Mills theory with 16 supercharges, PoS(LATTICE 2012) 064 [arXiv: 1212.4919] [INSPIRE].

[35] J. Nishimura, The origin of space-time as seen from matrix model simulations, Prog. Theor. Exp. Phys. 2012 (2012) 01A101 [arXiv:1205.6870] [InSPIRE].

[36] M. Hanada, Y. Hyakutake, G. Ishiki and J. Nishimura, Holographic description of a quantum black hole on a computer, Science 344 (2014) 882 [INSPIRE].

[37] M. Hanada, Does Yang-Mills theory describe quantum gravity?, arXiv:1407.5322 [INSPIRE].

[38] P.K. Townsend, The eleven-dimensional supermembrane revisited, Phys. Lett. B 350 (1995) 184 [hep-th/9501068] [INSPIRE].

[39] E. Witten, String theory dynamics in various dimensions, Nucl. Phys. B 443 (1995) 85 [hep-th/9503124] [INSPIRE].

[40] E. Cremmer, B. Julia and J. Scherk, Supergravity theory in eleven-dimensions, Phys. Lett. B 76 (1978) 409 [INSPIRE].

[41] M. Huq and M.A. Namazie, Kaluza-Klein supergravity in ten-dimensions, Class. Quant. Grav. 2 (1985) 293 [Erratum ibid. 2 (1985) 597] [INSPIRE].

[42] O. Aharony, S.S. Gubser, J.M. Maldacena, H. Ooguri and Y. Oz, Large-N field theories, string theory and gravity, Phys. Rept. 323 (2000) 183 [hep-th/9905111] [INSPIRE].

[43] N. Itzhaki, J.M. Maldacena, J. Sonnenschein and S. Yankielowicz, Supergravity and the large- $N$ limit of theories with sixteen supercharges, Phys. Rev. D 58 (1998) 046004 [hep-th/9802042] [INSPIRE].

[44] R. Kallosh and A. Rajaraman, Vacua of M-theory and string theory, Phys. Rev. D 58 (1998) 125003 [hep-th/9805041] [INSPIRE].

[45] M.B. Green, M. Gutperle and P. Vanhove, One loop in eleven-dimensions, Phys. Lett. B 409 (1997) 177 [hep-th/9706175] [INSPIRE].

[46] M.B. Green, H.-H. Kwon and P. Vanhove, Two loops in eleven-dimensions, Phys. Rev. D 61 (2000) 104010 [hep-th/9910055] [INSPIRE].

[47] M.B. Green, J.G. Russo and P. Vanhove, Non-renormalisation conditions in type-II string theory and maximal supergravity, JHEP 02 (2007) 099 [hep-th/0610299] [INSPIRE]. 
[48] R.M. Wald, Black hole entropy is the Noether charge, Phys. Rev. D 48 (1993) 3427 [gr-qc/9307038] [INSPIRE].

[49] V. Iyer and R.M. Wald, Some properties of Noether charge and a proposal for dynamical black hole entropy, Phys. Rev. D 50 (1994) 846 [gr-qc/9403028] [INSPIRE].

[50] Y. Hyakutake, Super Virasoro algebra from supergravity, Phys. Rev. D 87 (2013) 045028 [arXiv: 1211.3547] [INSPIRE]. 